Dal Laboratorio di Fisiologia della $R^{a}$ Università di Roma diretto dal prof. L. LucIanI.

\title{
Ricerche sulla differenziazione cellulare nello sviluppo embrionale.
}

Del

Dott. Amedeo Herlitzka.

Mit Tafel IV and 12 Figuren im Text.

Eingegangen am 17. August 1897.

\section{Introduzione.}

Ho voluto in queste ricerche seguire la differenziazione, che durante la vita embrionale subisce un gruppo di cellule, quelle dell'intestino, allo scopo di studiare, sotto quali condizioni esse si modifichino. A questo fine scelsi gli embrioni di smolge cristata e li studiai nei vari periodi dell' ontogenesi, limitando però le mie osservazioni fino al momento nel quale essi, uscendo dal muco che li circonda, vengono in rapporto più diretto con l'ambiente esterno. Perchè allora cessano di dominare prevalentemente quelle condizioni di sviluppo che sono insite alla natura dell' uovo, ed entrano in azione, come determinanti la differenziazione delle cellule e dei tessuti, tutti quei fattori del mondo esterno, che possono influire sull' organismo in via di sviluppo extraovarico, provocando specialmente gli adattamenti funzionali. Ed era mio obbiettivo, scindere nei loro elementi, per quanto il sommo viluppo dell' argomento lo consenta, le cause, per le quali la struttura di un gruppo di cellule varia nei diversi periodi della vita, astrazione fatta dai tanti e complicati stimoli funzionali, che possono fare sentire la loro azione sull' organismo, che si sta sviluppando.

Esaminai perciò in primo luogo, quali sono i cambiamenti che subisce la struttura della cellula; in secondo luogo come si comportino le sostanze immagazzinate nell' uovo, rispetto alle cellule stesse; 
in terzo luogo mi interessò la forma che assumono gli elementi cellulari ed i nuclei dell' intestino. Per ultimo studiai la grandezza degli elementi durante il differenziarsi dei medesimi.

Ricerche anteriori mi avevano fatto ritenere, che il volume della cellula e quello del nucleo dovessero avere un' importanza fondamentale per la vitalità dell' organismo. Infatti ho potuto vedere isolando $\mathrm{i}$ due blastomeri di uova di tritone dopo la prima segmentazione, che negli embrioni, cosi ottenuti da mezzo uovo solanto, gli elementi cellulari erano bensi in numero minore ehe negli embrioni normali, ma non avevano subíta nessuna modificazione nella loro grandezza.

Lo studio del volume degli elementi $-o$, per essere più precisi - del loro diametro, è stato fino ad oggi presso ehe esclusivamente condotto dal punto di vista morfologico: quanto alla sua importanza fisiologica essa non è stata quasi avvertita. Solo un recente studio di Lugaro riguarda le modificazioni che il volume della cellula nervosa subisce nei diversi stati funzionali: se questo aumento di volume durante la funzione, sia dovuto a modificazione della struttura cellulare, o ad accumulo di prodotti regressivi del ricambio, non è ancora perfettamente dimostrato.

Quanto al resto, l'importanza fisiologica del volume delle cellule è stata studiata solo per la funzione respiratoria dei corpuscoli rossi, per una funzione cioè, che non è legata alla struttura ed alla vitalità dell' elemento stesso, ma bensi alla composizione ed alle proprietà chimiche di una sostanza in esso accumulata.

Questa scarsezza delle ricerche sulla grandezza degli elementi cellulari, in rapporto alla loro funzionalità, credo giustifichi sufficientemente questo mio breve studio.

Esso è stato compiuto nel modo seguente.

Gli embrioni, ottenuti per mezzo della fecondazione artificiale, sono stati preparati per l'esame microscopico colla fissazione in sublimato corrosivo e colla susseguente colorazione nella miscela BIONDI, nella tionina, nell' emallume ed eosina, ecc., e colla fissazione nel liquido di Hermann, per la colorazione dei granuli fucsinofili col metodo di GaLEOTTI. Le misurazioni furono fatte disegnando su carta millimetrata i nuclei e le cellule mediante la camera lucida. Su questi disegni furono misurati il diametro massimo di ciascun elemento, ed il diametro maggiore fra quelli normali al primo, e ciò per conoscere le dimensioni dell' elemento ed i loro rapporti geometrici. Colla numerazione dei quadrati occupati da ciascun disegno si 
conosce l'area dei nuclei e delle cellule rispettive. Tenendo conto dei due diametri e dell' area si può arguire il volume delle cellule, se anche non ci è permesso di averne le dimensioni in cifre precise. Ma d'altra parte, difficoltà tecniche insuperabili, ei rendono impossibile aspirare per ora ad un' esattezza maggiore.

Nell' esporre le osservazioni fatte, mi atterrò alla massima brevità, specialmente nella descrizione della parte morfologica e strutturale, avendo questa per il mio scopo solo l'interesse di un controllo dell' avvenuta differenziazione.

Alla descrizione dei fatti osservati farò seguire la discussione dei risultati e le deduzioni sul loro valore per la fisiologia della cellula. $\mathrm{Ma}$, avendomi le mie conclusioni dato campo di fare alcune considerazioni generali sulle cause della differenziazione ontogenetica, in un secondo capitolo, dopo aver esposte e discusse le principali teorie che su tale argomento sono state emesse fino ad oggi, dirò brevemente di queste considerazioni e come esse aintino nell' interpretazione, ancora controversa, di alcuni fatti biologici.

Parte I.

Sul significato fisiologico della forma e della grandezza della cellula.

$\mathrm{I}^{0}$.

Le modificazioni della forma geometrica degli elementi intestinali durante la loro differenziazione istologica.

Le mie osservazioni sulla differenziazione delle cellule intestinali cominciano da quello stadio ontogenetico in cui, formato il tubo midollare, l'uovo comincia ad allungarsi ed assumere l'aspetto speciale dell' embrione. A quest' epoca l'intestino è costitutito da un cordone compatto, privo di lume, ed appare al microseopio, come un ammasso di un gran numero di granuli o zolle di dentoplasma, fra cui si trovano sparsi qua e là alcuni nuclei.

In questo stadio di sviluppo l'intestino permane per molti giorni: intanto a poco a poco, cominciando dall' estremo orale, si va formando il suo lume, limitato da pareti, che, da principio, presentano lo stesso aspetto dell' intestino dei primi gradi di sviluppo e poi si vanno differenziando come diremo più sotto.

La porzione dell' intestino ultima a subire queste modificazioni, è quella più anale: questa è ancora perfettamente indifferenziata al 
momento, nel quale gli embrioni escono dal muco, periodo a cui si arrestano le mie ricerche.

In una sezione d'intestino nei primi gradi di sviluppo (v. fig. 1), il fatto più noterole è la grande quantità di deutoplasma che vi si riscontra: questo in zolle più o meno grandi, quadrangolari od elittiche è circondato da un fine e delicato reticolo citoplasmatico a maglie larghe, che col metodo del Brondr si colora in una tinta rosso-violacea, mentre il deutoplasma assume un bel colorito rosso vivace. Limiti cellulari non vi si possono assolutamente riconoscere: se essi esistano e sieno mascherati dalle masse deutoplasmatiche, 0 se realmente il citoplasma non sia ancora distinto in cellule separate, non è possibile stabilire, nè vi sono dati per arguirlo. In mezzo alle maglie citoplasmatiche, e circondati da ogni parte da deutoplasma, stanno i nuclei, sparsi in tutto l'intestino in non grande quantità, un po' più abbondanti nella parte dorsale. Questi nuclei sono di forma varia, ora allungati, ora rotondeggianti, ora del tutto irregolari. In essi la sostanza cromatica si colora molto male coi colori basici; per usare il metodo del Biondi mi sono servito delle modalità indicate da Trambusti 1); ma non ebbi mai, in questo periodo di sviluppo i nuclei dell intestino colorati in verde, se non quando prolungai a 12 ore l'immersione delle sezioni in acido acetico, prima del trattamento colla miscela colorante, e quando portai la soluzione dell' acido acetico al 3 per mille. Ciò non ostante, come si osserva nella figura 1, la colorazione verde dei nuclei è poco intensa; con essa si mettono in evidenza solo poche zolle di cromatina, sparse qua e là nel nucleo e riunite tra loro da esili filamenti. La membrana nucleare è nettamente distinta, il nucleolo, unico, è ben colorato dalla sostanza acida. $\mathrm{Si}$ osservano in questo stadio rare eariocinesi, ed un numero abbastanza rilevante di segmentazioni dirette, in tutte le fasi.

Quanto alle dimensioni degli elementi, non posso riportare che quelle dei nuclei, perchè, come s'è detto, i limiti cellulari, se pure esistono, si sottraggono alla nostra osservazione.

I valori di queste dimensioni sono raccolti nella tabella $\mathrm{I}^{\mathrm{a}}$. In essa sono registrati i dati ottenuti dalla misurazione di 100 nuclei dell' intestino, in embrioni di 13 giorni. Nella prima colonna è riportata la grandezza dell' area di sezione dei nuclei in micromillimetri quadrati; nella seconda il diametro massimo in micromillimetri,

1) Trambusti, Contributo alla fisiopatologia della cellula epatica. Ricerche fatte nel laboratorio di anatomia normale di Roma ecc. 1895. 
nella terza il diametro maggiore fra quelli normali al precedente; nella quarta infine, il rapporto fra $\mathrm{i}$ due diametri, preso come 100 quello massimo.

Tabella? $\mathrm{I}^{\mathrm{a}}$.

\begin{tabular}{|c|c|c|c|c|c|c|c|c|c|c|c|c|c|c|c|c|c|c|c|}
\hline$\underset{\frac{\pi}{4}}{4}$ & 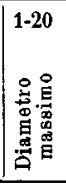 & 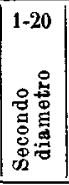 & 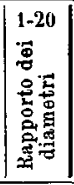 & $21-40$ & 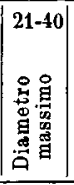 & 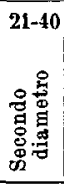 & 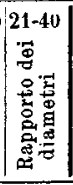 & $41-6$, & 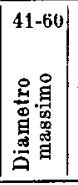 & 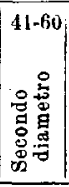 & 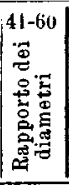 & $|61-80|$ & 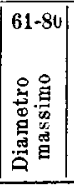 & 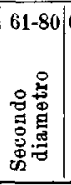 & 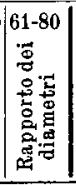 & $81-100$ & 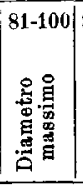 & 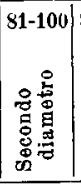 & 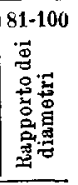 \\
\hline 52 & 9.5 & 9 & 94.5 & 55 & 10 & 9 & 90 & 49 & 11 & 7.5 & 68 & 45 & 10 & 6.5 & 65 & 71 & 11 & 9 & 81.5 \\
\hline 31 & 9.5 & 5 & 52.5 & 57 & 12 & 8 & 66.5 & 50 & 10 & 8 & 80 & .28 & 8 & 6 & 75 & 40 & 10 & 6 & 60 \\
\hline 24 & 9 & 6 & 66.5 & 53 & 12 & 7 & 58 & 35 & 9.5 & 6 & 63 & 52 & 11 & 7.5 & 68 & 39 & 9 & 6 & 66.5 \\
\hline 68 & 11.5 & 10 & 87 & 56 & 11 & 7.5 & 68 & 44 & 10 & 7 & 70 & 44 & 8.5 & 7 & 82 & 46 & 9 & 7.5 & 83 \\
\hline 36 & 11 & 6 & 54.5 & 74 & 10 & 9.5 & 95 & 51 & 11 & 8.5 & 77 & 40 & 9 & 8 & 89 & 61 & 10.5 & 9.5 & 90.5 \\
\hline 39 & 9.5 & 8.5 & 89.5 & 51 & 13 & 7.5 & 57.5 & 38 & 10 & 6.5 & 65 & 45 & 9 & 7 & 77.5 & 33 & 8 & 7.5 & 93.5 \\
\hline 40 & 11 & 5.5 & 50 & 78 & 12.5 & 10 & 80 & 48 & 11.5 & 7 & 61 & 52 & 10.5 & 8.5 & 81 & 57 & 10 & 8 & 80 \\
\hline 31 & 9 & 5.5 & 61 & 49 & 11 & 6.5 & 59 & 45 & 10 & 6.5 & 65 & 54 & 10.5 & 7.5 & 72 & 51 & 10.5 & 8.5 & 81 \\
\hline 59 & 10.5 & 9 & 85.5 & 49 & 9 & 8.5 & 94.5 & 44 & 9.5 & 6.5 & 68.5 & 30 & 9.5 & 7 & 73.5 & 35 & 8 & 6 & 75 \\
\hline 45 & 9.5 & 7.5 & 79 & 49 & 9.5 & $8 . \overline{5}$ & 89.5 & 51 & 10.5 & 8.5 & 81 & 50 & 10 & 8 & 80 & 40 & 9.5 & 8.5 & 89.5 \\
\hline 40 & 8.5 & 6 & 70.5 & 43 & 10 & 7 & 70 & 36 & 10 & 6 & 60 & 62 & 10 & 9.5 & 95 & 57 & 11 & 10 & 90.5 \\
\hline 26 & 8.5 & 4.5 & 53 & 41 & 10.5 & 6 & 57 & 51 & 10 & 8.5 & 85 & 52 & 110.5 & 6.5 & 62 & 57 & 10.5 & 10 & 93 \\
\hline 30 & 8.5 & 5.5 & 64.5 & 39 & 9 & 6.5 & 72 & 45 & 9.5 & 8 & 84 & 49 & 11 & 8 & 72.5 & 56 & 10.5 & 7.5 & 71.5 \\
\hline 34 & 9.5 & 5.5 & 58 & 39 & 9 & 7 & 77.5 & 60 & 10 & 8.5 & 85 & 37 & 9.5 & 6 & 63 & 57 & 10 & 8 & 80 \\
\hline 45 & 10 & 6.5 & 65 & 49 & 10 & 8 & 80 & 26 & 8 & 5 & 62.5 & 35 & 9 & 8 & 89 & 58 & 10.5 & 8.5 & 81 \\
\hline 66 & 11.5 & 8.5 & 74 & 34 & 8.5 & 6 & 70.5 & 49 & 10 & 8 & 80 & 50 & 10 & 8 & 80 & 58 & 9 & 8 & 89 \\
\hline 45 & 11 & 5.5 & 50 & 38 & 10.5 & 5.5 & 52.5 & 38 & 9.5 & 5.5 & 58 & 66 & 10 & 9 & 90 & 52 & 10.5 & 9 & 85.5 \\
\hline 37 & 10 & 6.5 & 65 & 44 & 11 & 7.5 & 68 & 49 & 9.5 & 7.5 & 79 & 52 & 9.5 & 8 & 84 & 54 & 10.5 & 8 & 76 \\
\hline 48 & 10.5 & 7 & 66.5 & 45 & 11 & 7 & 63.5 & 55 & 10 & 9.5 & 95 & 47 & 9 & 7.5 & 83 & 48 & 10 & 7 & 70 \\
\hline 66 & 13 & 8.5 & 73 & 39 & 9 & 6.5 & 72 & 51 & 10.5 & 6.5 & 62 & 66 & 11 & 9 & 81.5 & 72 & 10 & 9.5 & 95 \\
\hline
\end{tabular}

Come si vede da questa tabella la grandezza dell' area varia da 24 micromillimetri quadrati a 78 con una frequenza massima per i valori da 36 a 55 micro, limite che è occupato dal 65 per cento dei nuclei. I numeri molto bassi dipendono probabilmente dal fatto che, occupando ciascun nucleo più di una sezione (le sezioni avevano da 3 a $6 \mu$ di spessore), non tutti i nuclei venivano tagliati a livello del loro equatore, ma molti invece più 0 meno distanti da questo.

Il rapporto fra $\mathrm{i}$ due diametri oscilla in limiti molto ampi, ma non scende mai al disotto di 50, nè sale oltre 94. Nella massima parte dei nuclei però il rapporto è tra 58 e 70 e tra 78 e 82 .

La struttura degli elementi dell' intestino, più su descritta, non varia affatto per molti giorni, e le dimensioni dei nuclei rimangono 
immutate, come lo dimostrarono le numerose misurazioni eseguite che qui per brevità tralascio di riportare. Poi man mano, mentre diminuisce la quantità del deutoplasma, comincia a modificarsi la struttura degli elementi intestinali, e - fatto importante - variano contemporaneamente le dimensioni nucleari ').

Questa differenziazione è iniziata, come si è detto, da una diminuzione della quantità del deutoplasma per cui il reticolo citoplasmatico si fa più evidente: esso verso il lume intestinale, che si è andato intanto formando, finisce con un margine ben distinto, che si colora intensamente, coi colori acidi (vedi fig. 2 e 3).

Dobbiamo, però, qui distinguere due forme di differenziazione in questo stadio.

Nella prima (fig. 2) vediamo la struttura del citoplasma variare dal suo limite esterno al lume intestinale. Esternamente esso è costituito da un sottile e delicato reticolo, a maglie piccolissime; in questo si trovano i nuclei, di cui parleremo fra poco. Al di là dei nuclei, il citoplasma circonda i granuli deutoplasmatici, dividendosi in rami più o meno robusti, paralleli fra di loro, e diretti nella direzione dei raggi dell' intestino: essi sono congiunti tra loro da trabecole trasversali. Tra $\mathrm{i}$ granuli di deutoplasma ed il citoplasma vi ha un alone incolore, sulla cui natura nulla ei è possibile di dire. I rami citoplasmatici poi sembrano esser costituiti prevalentemente da fibrille decorrenti nella loro stessa direzione. Presso il lume intestinale, il citoplasma appare formato da un reticolo di maglie meno sottili, che non nella sua parte esterna: esse vengono limitate verso il lume da una specie di cuticula, molto intensamente colorata.

Il deutoplasma sta fra questa cuticula ed i nuclei, in mezzo agli spazi lasciati liberi dai rami citoplasmatici già descritti; soltanto pochi granuli si riscontrano in mezzo ai nuclei stessi, e fra questi ed il margine esterno dell' intestino. Questa localizzazione si va accentuando tanto più, quanto maggiore è la differenziazione. I granuli di deutoplasma sono, in questo periodo, in generale più piccoli di quelli del periodo precedente, e sono quasi tatti di forma elittica; essi sono collocati, quasi esclusivamente, col loro massimo diametro nella direzione del raggio dell' intestino, o sono di poco inclinati su questo.

1) La descrizione delle modificazioni dell' intestino non si riferisce alla parte cefalica di questo, non essendo stata essa studiata, ma alla parte a questa seguente. 
In questa stessa direzione sta il diametro massimo dei nuclei. Questi sono disposti l'uno accanto all' altro, più o meno vicini al limite esterno dell intestino, in mezzo al fine reticolo citoplasmatico. La loro forma è allungata, e sono piuttosto voluminosi. La cromatina è più abbondante che nel primo periodo di sviluppo, o, per esser più esatti, se ne può mettere in evidenza una maggiore quantità coi colori basici, coi quali si colora più facilmente, non però quanto $i$ nuclei degli altri tessuti (sistema nervoso ed ectoderma). In questi nuclei i nucleoli sono quasi costantemente due.

La fig. 3, ci mostra l'altra forma di differenziazione, che troviamo in questo periodo di sviluppo. In essa il reticolo citoplasmatico è a maglie ampie: il deutoplasma più abbondante, a zolle più grosse, è sparso, senza ordine dimostrabile, in mezzo al citoplasma. I nuclei attraversano l'intestino, disposti l'uno accanto all' altro in lunghe serie. Essi sono o poligonali o rotondeggianti. Anche in essi, come in quelli or. ora descritti, si è resa più evidente la cromatina, mediante il verde di metilene: ma, al contrario che in quelli, il nucleolo è in essi sempre unico. Anche qui gli elementi sono limitati verso il lume intestinale da una cuticola, che però è molto meno evidente che nell' altro caso.

In nessuna delle due forme di differenziazione è possibile riconoscere un limite fra cellula e cellula; e forse, per la speciale disposizione dei nuclei, che sono strettamente addossati l'uno all' altro (vedi fig. 3), è lecito dubitare, che qui il citoplasma sia morfologicamente individualizzato in tante cellule, quanti sono $i$ nuclei.

Mi sono dovuto perciò limitare ad esaminare le dimensioni dei soli nuclei. I dati ricavati da 100 nuclei in queste misurazioni sono raccolti nelle due tabelle II e III.

La tabella II contiene quelli che si riferiscono ai nuclei prima descritti e corrispondenti alla fig. 2, la tabella III quelli dei nuclei che per ultimi ci occuparono, e che sono riprodotti nella fig. 3 .

Altre misurazioni fatte su vari embrioni, che presentavano le stesse differenziazioni nei vari giorni di sviluppo, mostrano dati perfettamente corrispondenti a questi che qui riportiamo.

Le cifre sono disposte come nella tabella I. 
Tabella II.

\begin{tabular}{|c|c|c|c|c|c|c|c|c|c|c|c|c|c|c|c|c|c|c|c|}
\hline 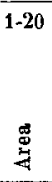 & 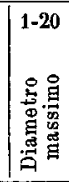 & 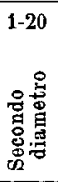 & 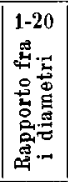 & 岛 & 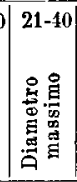 & 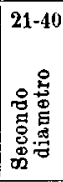 & 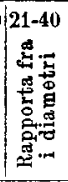 & 离 & 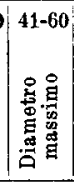 & 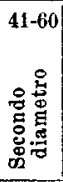 & 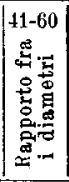 & 焉 & 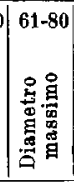 & 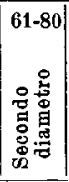 & 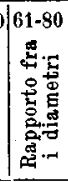 & 惫 & 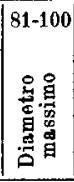 & 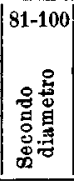 & s \\
\hline 43 & 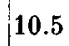 & 5 & 47.5 & 40 & 11 & 5 & 45.5 & 50 & 12 & 6 & 50 & 49 & 12 & 6 & 50 & 76 & 13.5 & 6.5 & \\
\hline 60 & & 6 & 4 & 48 & 12 & 5 & 40 & 48 & 1 & 0 & 54.5 & 45 & 12 & 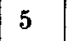 & 41.5 & 62 & 13 & 6.5 & 2 \\
\hline 41 & 1 & 5 & 41.5 & 50 & 11 & 6 & 54.5 & 38 & 1 & 5 & 45.5 & 57 & 14 & 6 & & 4 & 1 & & \\
\hline 42 & 1 & 5 & 45.5 & 30 & 11 & 5 & 45.5 & 50 & 1 & 6 & 50 & 62 & 13 & & $\mathbf{4}^{\mathbf{4}}$ & 58 & 10 & & 6 \\
\hline 64 & & 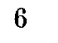 & 4 & 28 & do & 4 & 40 & 44 & & 5.5 & 48 & 65 & 12.5 & 6.5 & 5 & 5 & 11.5 & & \\
\hline 58 & & 6.5 & 5 & 76 & 1 & 6.5 & 50 & 1 & 1 & - & 50 & 0 & 9.5 & 4.5 & 4 & 45 & 11 & & \\
\hline 54 & & 5.5 & & & 1 & 4.5 & 45 & 8 & & 6 & 10 & 34 & 10 & & 50 & 33 & 0 & & \\
\hline 73 & d. & 7 & 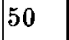 & 4 & & 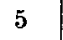 & & 37 & & 5.5 & & 7 & 1 & 5 & 4 & & & & \\
\hline 68 & 1 & 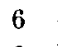 & 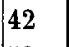 & $i$ & & 6.5 & & 49 & & 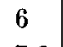 & 54.5 & 2 & 1 & 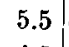 & 5 & 47 & & & \\
\hline 53 & 1.5 & 6 & 5 & 79 & 14 & 7.5 & 53.5 & 71 & & 7.5 & 57.5 & 7 & 1 & 5 & 4 & 6 & 12 & & \\
\hline 39 & 11 & 4.5 & & 5 & & 6 & 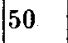 & t & & 7 & $d=$ & & 11 & 5 & 50 & 52 & 12 & 5 & \\
\hline 31 & 9.5 & 4 & , & 5 & 12 & 6 & $I_{5}$ & & & 5 & & 3 & 12 & & 5 & 3 & & & \\
\hline 46 & 11 & 5. & 5 & 3 & 9. & 4. & 4 & 4 & & 5 & .5 & 7 & 1 & & 4 & 47 & 1 & & \\
\hline 36 & 9. & 5 & 5 & 53 & 1 & 5.5 & 48 & 68 & 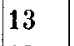 & 6.5 & & 50 & 1 & 5 & $1^{4}$ & 8 & 1 & & \\
\hline 60 & 13. & 6 & & 7 & & 7 & 52 & 45 & 1 & 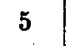 & 41.5 & 45 & 11.5 & 5.5 & 4 & 31 & 10 & 0 & \\
\hline 64 & 13 & 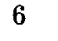 & do & 3 & 1 & 5 & 50 & 47 & 1 & 5 & 4 & 37 & 10 & 4.5 & 4 & 17 & 11.5 & .5 & \\
\hline 70 & 13 & 6 & 4 & 6 & 13 & 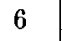 & 4 & 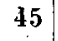 & 12 & 5.5 & 4 & & & 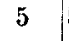 & 52 & 54. & 11.5 & & 2 \\
\hline 65 & 12 & 6. & jo & 6 & 1 & 0 & D & 3 & 12 & 5.5 & 4 & 42 & 11.5 & 8 & 52 & 40 & 1 & & \\
\hline 60 & & 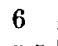 & & 5 & it & 5. & 18 & & 9 & 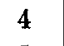 & 44.3 & 55 & & 5.5 & & ग3 & 10. & & \\
\hline 54 & 13.5 & 5. & 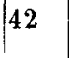 & 53 & 1 & $c$ & 48 & 36 & 10 & 5 & 50 & 66 & 13.5 & $c$ & 44.5 & 54 & 13 & $\mathbf{0}$ & \\
\hline
\end{tabular}

In queste tabelle si osserva che l'area è in complesso un po' maggiore nei nuclei corrispondenti alla differenziazione prima descritta, che non in quelli che sono stati riprodotti nella figura 3 della tavola.

Differenze maggiori, e molto più importanti, troviamo nei rapporti fra i diametri. Ciò si osserva, meglio che nelle tabelle, nel diagramma della figura $b$, dove sono espressi graficamente i dati ottenuti dalle misurazioni eseguite.

I numeri dell' ascissa indicano in questa figura il rapporto fra i due diametri, quelli dell' ordinata la percentuale dei nuclei per ciascun rapporto. Le aste più chiare corrispondono alla tabella II, quelle più scure alla tabella III. Le prime vanno da un rapporto minimo di 40 ad uno massimo di 58, raggiungendo fra 46 e 50 il punto più alto sull ordinata. La seconda curva ha un percorso molto diverso, estendendosi sull' ascissa da 62 a 100 , mentre arriva al suo fastigio in corrispondenza di 89 . 
Tabella III.

\begin{tabular}{|c|c|c|c|c|c|c|c|c|c|c|c|c|c|c|c|c|c|c|c|}
\hline 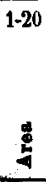 & 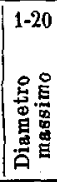 & 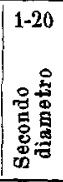 & 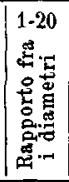 & 趌 & 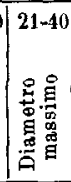 & 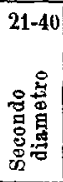 & 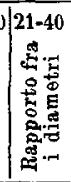 & $41-60$ & 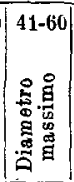 & 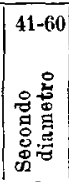 & 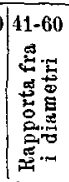 & $|61-80|$ & 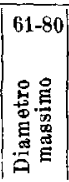 & 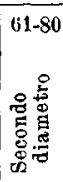 & 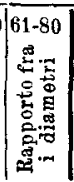 & $81-100$ & 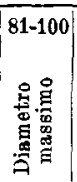 & 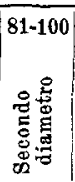 & 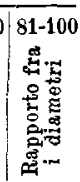 \\
\hline 52 & 10 & 7 & 70 & 37 & 8 & 6.5 & 81 & 32 & 8.5 & 5.5 & 64.5 & 53 & 9 & 8 & 89 & 46 & 8 & 6.5 & 81 \\
\hline 50 & 8 & 7 & 87.5 & 42 & 9 & 6.5 & 72 & 34 & 7.5 & 6 & 80 & 47 & 8.5 & 8 & 94 & 35 & 6.5 & 6 & 92 \\
\hline 33 & 7.5 & 6 & 80 & 42 & 8 & 7 & 87.5 & 45 & 8 & 7 & 87.5 & 43 & 8.5 & 7 & 82 & 41 & 7 & 6 & 85.5 \\
\hline 56 & 10 & 7.5 & 75 & 50 & 9 & 8 & 89 & 55 & 8.5 & 7 & 82 & 35 & 7.5 & 6 & 80 & 41 & 8 & 6.5 & 81 \\
\hline 47 & 8 & 7 & 87.5 & 60 & 9 & 8 & 89 & 51 & 9 & 7 & 78 & 30 & 7 & 6 & 85.5 & 60 & 9 & 8.5 & 94.5 \\
\hline 55 & 9 & 7 & 78 & 42 & 9 & 6.5 & 72 & 38 & 8 & 6 & 75 & 37 & 8 & 6 & 75 & 48 & 9 & 7 & 78 \\
\hline 32 & 8 & 5 & 62.5 & 60 & 10 & 8 & 80 & 31 & 7 & 5.5 & 78.5 & 50 & 9 & 8 & 89 & 42 & 8 & 6 & 75 \\
\hline 61 & 10 & 7 & 70 & 58 & 10 & 7 & 70 & 30 & 7 & 6 & 85.5 & 45 & 8 & 7 & 87.5 & 43 & 8 & 7 & 87.5 \\
\hline 45 & 8 & 7.5 & 97.5 & 36 & 7 & $l$ & 100 & 44 & 8 & 6 & 75 & 47 & 9.5 & 7 & 73.5 & 37 & 8 & 6 & 75 \\
\hline 37 & 8.5 & 6 & 70.5 & 50 & 9 & 7.5 & 83.5 & 43 & 8 & 6 & 75 & 41 & 8.5 & 6 & 70 & 44 & 8.5 & 7 & 82 \\
\hline 43 & 8 & 7 & 87.5 & 50 & 9.5 & 7 & 73.5 & 41 & 8 & 6 & 75 & 43 & 8 & 7 & 87.5 & 46 & 8 & . & 87.5 \\
\hline 36 & 8 & 6 & 75 & 50 & 8 & 7 & 87.5 & 48 & 8 & 7 & 87.5 & 47 & 7 & 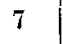 & 100 & 40 & 8.5 & 6 & 70.5 \\
\hline 26 & 6 & 6 & 100 & 42 & 9 & 6 & 66.5 & 70 & 10.5 & 8 & 76 & 37 & 7 & 6.5 & 92.5 & 49 & 9 & 7 & 78 \\
\hline 35 & 8.5 & 6 & 70.5 & 36 & 7 & 5.5 & 78.5 & 54 & . & 7 & 78 & 40 & 9 & 6.5 & 72 & 41 & 8 & 7 & 87.5 \\
\hline 49 & 9 & 8 & 89 & 41 & 8.5 & 6 & 70.5 & 50 & 10 & 7 & 70 & 46 & 8 & $\tau$ & 87.5 & 44 & 8 & 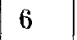 & 75 \\
\hline 31 & 7 & 6 & 85.5 & 49 & 9.5 & 7 & 73.5 & 41 & 7 & 6 & 85.5 & 62 & 9 & 8 & 189 & 43 & 8 & 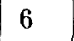 & 75 \\
\hline 43 & 8 & 7 & 87.5 & 50 & 10 & 7 & 70 & 40 & 8 & 6 & 75 & 51 & 9 & 7 & 78 & 31 & 7 & 6 & 85.5 \\
\hline 30 & 7 & 5.5 & 78.5 & 46 & 8 & 7 & 87.5 & 48 & 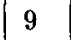 & 7 & 78 & 42 & 8 & 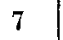 & 87.5 & 41 & 8 & 6.5 & 92.5 \\
\hline 36 & 7.5 & 6.5 & 86.5 & 36 & 7 & 5.5 & 78.5 & 48 & 9 & 7 & 78 & 32 & 7 & 6 & 85.5 & 36 & 7.5 & 6 & 80 \\
\hline 42 & 8 & 7 & 87.5 & 51 & 9.5 & ז & 73.5 & 61 & 10 & 8.5 & 85 & 43 & . 7.5 & 6.5 & 86.5 & 48 & 8 & 7 & 87.5 \\
\hline
\end{tabular}

I rapporti geometrici delle due specie di nuclei sono molto diversi; mentre $\mathrm{i}$ primi, in sezione, si avvicinano alla figura di un' elissi molto allungata, i secondi tendono ad una forma molto più rotondeggiante; i due diametri principali di questi ultimi sono molto più simili fra di loro che nel primo caso.

Date quindi le aree pressochè eguali nei due ordini di nuclei, coi rapporti geometrici dei diametri cosi diversi, e considerato che il diametro della terza dimensione è presso a poco eguale al minore dei diametri misurati, dovremo concludere, che anche i volumi dei nuclei, il rapporto fra volume e superficie, e tanto più la posizione dei vari elementi della massa nucleare, rispetto alla superficie del nucleo, devono essere differenti.

Dobbiamo ricordare inoltre ehe, sebbene la posizione dei diversi elementi della massa del nucleo, rispetto alla superficie di questo, varii col variare del rapporto fra quest' ultima ed il volume, pure queste due modificazioni non si verificano nella medesima proporzione, e la 
prima può avvenire, anche indipendentemente dalla seconda, per un cambiamento di forma del nucleo, non legato a mutamenti nè del volume, nè della superficie dell' elemento.

Ma ancor più interessanti diventano questi dati, se confrontiamo le due curve di cui fino a ora ci siamo occupati, con una dei rapporti fra $i$ diametri dei nuclei non ancora differenziati, come sono quelli

Fig. $a$.

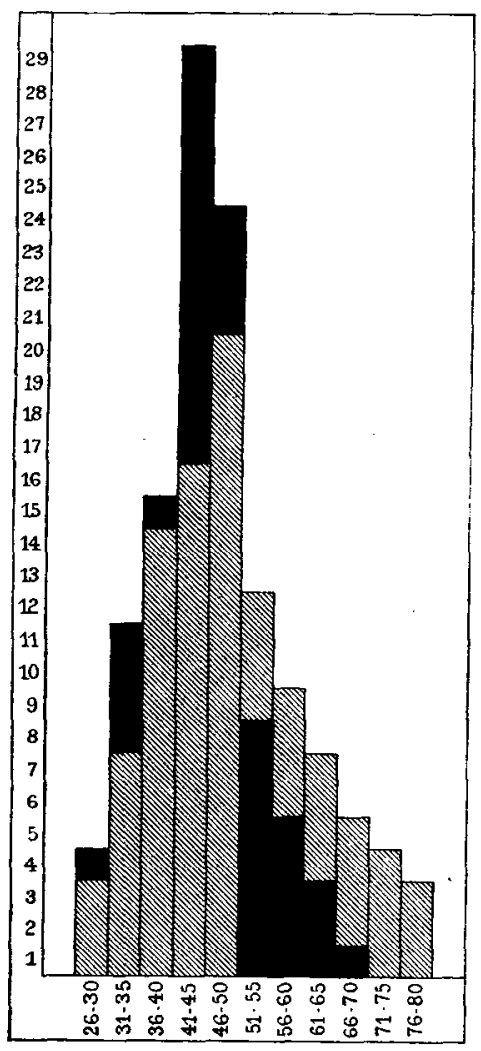

della fig. 1. Nel diagramma della fig. $b$ questa curva è rappresentata dalle aste in bianco. Essa occupa un tratto molto ampio dell' ascissa e mostra un percorso molto irregolare. La sua posizione è quasi intermedia fra le altre due curve. E poichè questi nuclei indifferenziati, rappresentano appunto l'origine comune, da cui si sono poi differenziate tutte due le specie di nuclei di questo secondo stadio di sviluppo, potremo dire che $\mathrm{i}$ rapporti geometrici di questi ultimi, cioè la forma della loro sezione, i rapporti fra volume e superficie, ecc. si sono modificati in senso divergente, partendo da un substrato comune.

La fig. a rappresenta il diagramma della grandezza delle aree di queste due serie di nuclei. In essa i numeri della ascissa danno la grandezza delle aree in micromillimetri quadrati. Tutte le altre indicazioni sono eguali a quelle della figura $b$.

In uno stadio ulteriore di sviluppo, troviamo infine il citoplasma diviso in cellule, e queste distinte in due specie: cellule di rivestimento e cellule glandolari. Ciò avviene pochi giorni prima che l'embrione esca dal suo muco.

Le cellule di rivestimento (figg. 4 e 5) si presentano molto allungate di forma cilindrica. Il citoplasma è molto delicatamente reticolato non però in modo omogeneo; verso il lume intestinale esso si va facendo sempre più compatto, e presenta al margine una cuti- 
cola fortemente colorata, che appare come l'ultimo di vari strati citoplasmatici paralleli fra loro ed al limite cellulare, i quali diventano sempre più densi, quanto più si avvicinano a quest' ultimo.

Questa cuticola porta in alcuni tratti (fig. 4) delle sottilissime ciglia che sporgono nell' interno del lume intestinale: in altre cellule mancano queste ciglia. Il deutoplasma è molto scarso e solo si

Fig. $b$.

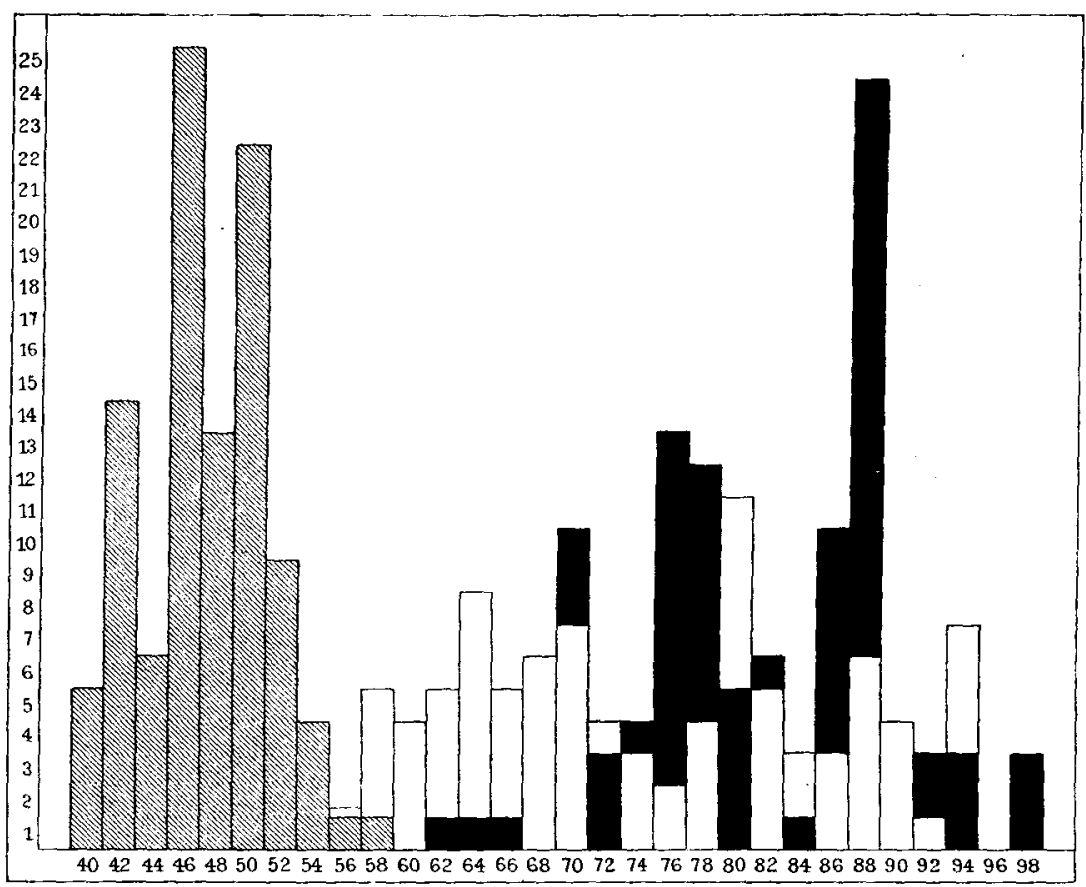

scorgono rare zolle di questo, in prevalenza fra il nucleo e la cuticola, in pochi casi fra i nuclei stessi o fra questi ed il limite esterno della cellula. In vicinanza di quest' ultimo si trovano i nuclei. Essi presentano zolle di cromatina molto più evidenti ed abbondanti che negli stadi anteriori. I nucleoli sono quasi sempre in numero di due per ciascun nucleo: alcune eccezioni si possono facilmente spiegare col fatto, che uno dei nucleoli sia rimasto fuori della sezione in esame.

Le cellule glandolari (fig. 6) sono di forma trapezoide, col lato più piccolo in corrispondenza del lume glandolare. II citoplasma in esse ha un aspetto di delicatissimo reticolo omogeneo: non presenta 
traccie di cuticola. Anche in queste cellule il deutoplasma è scarso, quanto in quelle di rivestimento. I nuclei rotondeggianti o poligonali sono disposti al limite esterno della cellula, restando cosi tra essi ed il lume glandolare un' ampia zona di citoplasma. La sostanza cromatica è qui più abbondante ed evidente che in tutti gli altri nuclei descritti: essa è disposta in zolle e filamenti considerevoli.

Il nucleolo è sempre unico.

Le dimensioni di cento cellule di rivestimento sono date dalla tabella IV, quelle di altrettante cellule glandolari dalla tabella V. Le tabelle VI e VII si riferiscono ai rispettivi nuclei, la VI, cioè a quelli di rivestimento, la settima a quelli glandolari.

Tabella IV.

\begin{tabular}{|c|c|c|c|c|c|c|c|c|c|c|c|c|c|c|c|c|c|c|c|}
\hline 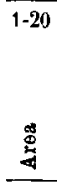 & 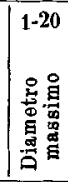 & 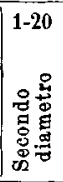 & 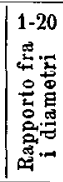 & $|21-40|$ & 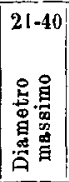 & 迹 & 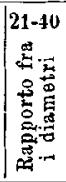 & $\stackrel{\varpi}{4}$ & 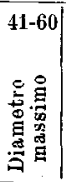 & 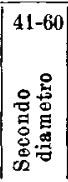 & 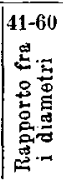 & 苞 & 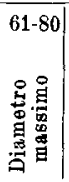 & 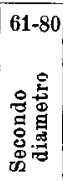 & 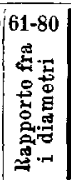 & $\mid 81-100$ & 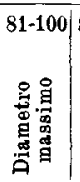 & 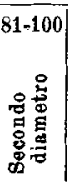 & 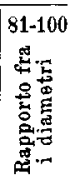 \\
\hline 18 & & 6.5 & 34 & 102 & & 5 & 21 & 160 & 25 & 7.5 & 30 & 90 & 22 & 5 & 22.5 & 75 & 16 & 5 & 31 \\
\hline 76 & 1 & 5.5 & 30 & & 21 & 4.5 & 21.5 & 109 & 23 & 5.5 & 24 & 111 & 22 & 5.5 & 25 & 82 & 19 & $\mathrm{~J}$ & 26 \\
\hline$\$ 14$ & 20 & 7 & 35 & 118 & & 5.5 & 27.5 & 107 & 23 & 5 & 21.5 & 84 & 21 & 4.5 & 21.5 & 84 & 19 & 5 & 26 \\
\hline 60 & 1 & $4 . \check{\partial}$ & 23.5 & 8 & 23 & 4.5 & 20.5 & 83 & 23 & 4.5 & 20.5 & 112 & 23.5 & 5 & 21 & 115 & 21 & 5.5 & 26 \\
\hline 84 & 2 & 5 & 23.5 & 122 & & 7 & 31.5 & 110 & 23 & 5 & 21.5 & 93 & 22 & 5 & 22.5 & 79 & 19 & 4 & 22 \\
\hline 164 & & 7.5 & 32 & 100 & 22 & 5.5 & 125 & 93 & 22 & 5 & 22.5 & 105 & 20.5 & 6 & 29 & 119 & 21.5 & .5 & 25.5 \\
\hline 138 & 22.5 & 7 & 31 & 5 & 23. & 5.5 & 23.5 & 94 & 21 & 5 & 23.5 & 71 & 20.5 & 4 & 19.5 & 114 & 22.5 & 5 & 2 \\
\hline 3 & 2 & 8 & 38 & 8 & 25 & 7.5 & 30 & 80 & 20 & 5 & 25 & 88 & 19 & 5 & 25.5 & 124 & 21 & & 28.5 \\
\hline 0 & 2 & 6 & 28.5 & 140 & & 7 & 30.5 & 97 & 22 & 5 & 22.5 & 170 & 23 & 7.5 & 32 & 120 & 21 & 6 & 28.5 \\
\hline 3 & 2 & 6.5 & 30 & & 21 & 6 & 28.5 & 146 & 24 & 7.5 & 23 & 137 & 25 & 6 & 24 & 21 & 21 & & 28.5 \\
\hline 22 & 2 & 6.5 & 28 & & 18 & 4.5 & 25 & 110 & 21 & 6 & 28.5 & 158 & 25 & 7 & 27.5 & 142 & 22 & & 31.5 \\
\hline 1 & 23. & 6 & 25.5 & 89 & 18. & 5.5 & 30.5 & 80 & 21 & 4 & 18 & 133 & 23.5 & 6.5 & $2 \overline{7} .5$ & 139 & 22.5 & & 1 \\
\hline 116 & 2 & 6 & 26 & 7 & & 5 & 27.5 & 117 & 19.5 & o & 30.5 & 136 & 26 & 6 & 23 & 105 & 22 & 5 & 22.5 \\
\hline 46 & 2 & 7 & 32.5 & 7 & 21 & 5 & 23.5 & 113 & 21 & 5.5 & 26 & 124 & 23.5 & 6 & 25.5 & 99 & 22 & & 22.5 \\
\hline 89 & o & 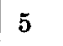 & 22.5 & 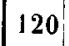 & 26 & 7.5 & 29 & 110 & 24 & & 21 & & 25 & - & 28 & 116 & 24 & i) & 23 \\
\hline 110 & 15 & 6 & 28.5 & 107 & & 6.5 & 26.5 & 123 & 21 & 0 & 28.5 & 7 & 23 & 1. & 30.5 & 78 & 20 & & - \\
\hline 111 & 2 & 6 & 28.5 & 105 & & 5.5 & 24 & 125 & 23 & 5.5 & 24 & 116 & 21 & 6 & 28.5 & 105 & 21 & 5.5 & 26 \\
\hline 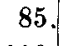 & & 4.5 & 2 & 6 & & 5 & 22.5 & 1 & 24 & 6.5 & 26. & 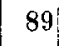 & 20 & 5 & 2 & 109 & 22 & o & 22 \\
\hline & 12 & 5.5 & 23.5 & 106 & & 5.5 & 26 & 130 & 25 & 5.5 & 22 & 60 & 17 & 3.5 & 20.5 & 97 & 19 & 5.5 & 29 \\
\hline 134 & 24.5 & 6.5 & 26.5 & & & 5 & 22.5 & 123 & 23.5 & 5.5 & 23.5 & 67 & 15 & 5 & 33 & 73 & 17.5 & 5.5 & 31.5 \\
\hline
\end{tabular}


Tabella V.

\begin{tabular}{|c|c|c|c|c|c|c|c|c|c|c|c|c|c|c|c|c|c|c|c|}
\hline 匙 & 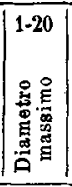 & 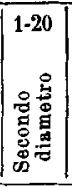 & 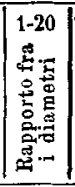 & 离 & 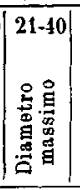 & 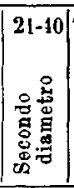 & 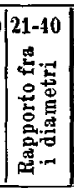 & $\stackrel{\leftrightarrows}{4}$ & 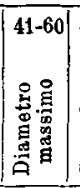 & 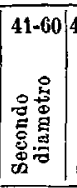 & 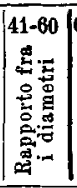 & 离 & 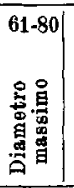 & 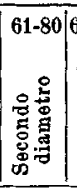 & 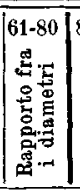 & $|81-100|$ & 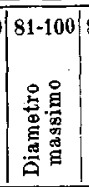 & 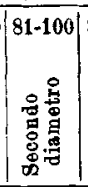 & 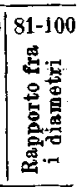 \\
\hline 65 & 16 & 13.5 & 84.5 & 127 & 15 & 11 & 73 & 107 & 14 & 9 & 64 & 77 & 10 & 9 & 90 & 140 & 16 & 12 & 75 \\
\hline 148 & 17 & 13 & 76.5 & 124 & 13 & 12 & 92 & 75 & 11 & 8.5 & 77 & 95 & 12 & 10.5 & 87.5 & 124 & 13.5 & 11.5 & 85 \\
\hline 127 & 13 & 12.5 & 96 & 93 & 13.5 & 9 & 66.5 & 110 & 13.5 & 11.5 & 85 & 84 & 12 & 9 & 75 & 124 & 13.5 & 12 & 89 \\
\hline 94 & 12.5 & 12 & 96 & 88 & 11.5 & 8.5 & 73.5 & 95 & 13.5 & 10.5 & 77.5 & 115 & 13.5 & 12 & 89 & 89 & 11 & 10 & 91 \\
\hline 129 & 15 & 11 & 73 & 95 & 12 & 9 & 75 & 94 & 12 & 9 & 75 & 109 & 13 & 12 & $\dot{9}_{2}$ & 98 & 13 & 9.5 & 73 \\
\hline 104 & 13 & 10.5 & 80.5 & 97 & 15 & 10.5 & 70 & 112 & 12 & 10 & 83 & 103 & 13.5 & 10 & 74 & 93 & 11 & 10 & 91 \\
\hline 168 & 18 & 13 & 72 & 71 & 11 & 9 & 81.5 & 74 & 12.5 & 8 & 64 & 96 & 11 & 10 & 91 & 89 & 11 & 10 & 91 \\
\hline 85 & 13 & 9 & 69 & 115 & 12.5 & 12 & 96 & 71 & 11 & 8 & 72.5 & 106 & 11.5 & 10.5 & 91 & 96 & 11 & 10.5 & 95.5 \\
\hline 116 & 15 & 10 & 66.5 & 103 & 11.5 & 9.5 & 582.5 & 70 & 11 & 8 & 72.5 & 114 & 11.5 & 11 & 95.5 & 83 & 11 & 8.5 & $7 i$ \\
\hline 116 & 16 & 9 & 56 & 117 & 13 & 11.5 & 88.5 & 83 & 11 & 10.5 & 95.5 & 87 & 11.5 & 9.5 & 82.5 & 80 & 11.5 & 9.5 & 82.5 \\
\hline 99 & 14 & 10 & 71.5 & 143 & 14.5 & 13 & 89.5 & 82 & 11 & 10.5 & 95.5 & 115 & 13 & 10 & 77 & 89 & 10 & 10 & 100 \\
\hline 108 & 12.5 & 12 & 96 & 78 & 10.5 & 10 & 100 & 73 & 11 & 8.5 & 73.5 & 80 & 12 & 9 & 75 & 85 & 12 & 9 & 75 \\
\hline 120 & 14 & 12 & 85.5 & 90 & 12.5 & 9.5 & 76 & 116 & 13.5 & 12 & 92 & 74 & 11 & 8.5 & 77 & 66 & 10 & 8.5 & 85 \\
\hline 100 & 13.5 & 11 & 81.5 & 101 & 12 & 10 & 83 & 192 & 14.5 & 12.5 & 86 & 83 & 12 & 8 & 66.5 & 101 & 12 & 10 & 83.5 \\
\hline 112 & 13 & 11.5 & 88.5 & 113 & 14.5 & 11 & 78.5 & 112 & 13 & 11.5 & 88.5 & 73 & 10.5 & 9 & 85.5 & 78 & 11 & 10 & 91 \\
\hline 96 & 12 & 11 & 91.5 & 135 & 16.5 & 12 & 72.5 & 105 & 11 & 10 & 91 & 52 & 10 & 7 & 70 & 96 & 12 & 9.5 & 79 \\
\hline 104 & 12.5 & 11 & 88 & 97 & 14 & 9 & 64 & 70 & 11 & 8 & 72.5 & 67 & 10 & 8 & 80 & 113 & 12.5 & 10 & 80 \\
\hline 118 & 15 & 11 & 73 & 106 & 13 & 12 & 92 & 105 & 13 & 10 & 77 & 65 & 10 & 8 & 80 & 87 & 11 & 9 & 81.5 \\
\hline 85 & 12 & 11 & 91.5 & 136 & 15 & 14 & 93 & 150 & 15 & $13 / 8$ & 86.5 & 69 & 10 & 8.5 & & 101 & 13 & 10 & 77 \\
\hline 84 & 13 & 10 & 77 & 106 & 16 & 9 & 56 & & 13.5 & & 70.5 & & & & 70.5 & 102 & 15 & 13 & 86.5 \\
\hline \multicolumn{20}{|c|}{ Tabella VI. } \\
\hline
\end{tabular}

\begin{tabular}{|c|c|c|c|c|c|c|c|c|c|c|c|c|c|c|c|c|c|c|c|}
\hline 趈 & 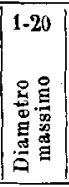 & 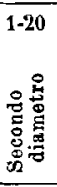 & 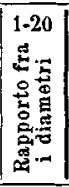 & 离 & 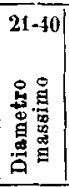 & 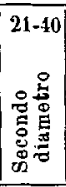 & 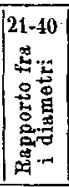 & $41-60$ & 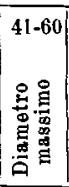 & 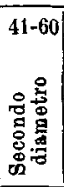 & 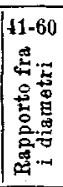 & 馬 & 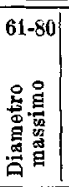 & 竎 & 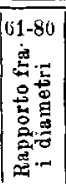 & 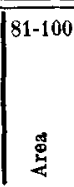 & 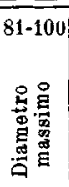 & 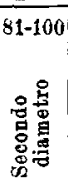 & 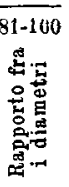 \\
\hline 51 & 12 & 5 & 41.5 & 58 & 12.5 & 5 & 40 & 51 & 13 & 5 & 38.5 & 53 & 12 & 5 & 41.5 & 58 & 13.5 & 5 & 37 \\
\hline 30 & 11 & 4 & 36.5 & 40 & 11 & 4.5 & 11 & 43 & 14 & 4.5 & 32 & 60 & 12.5 & 5 & 40 & 51 & 12 & 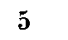 & 41.5 \\
\hline 35 & 12 & 4 & 33 & 69 & 15.5 & 5.5 & 35.5 & 44 & 11.5 & 5 & 43.5 & 51 & 13 & D & 38.5 & 56 & 14.5 & 5.5 & 38 \\
\hline 62 & 15.5 & 5 & 32 & 72 & 15 & 5.5 & 36.5 & 36 & 13 & 5 & 38.5 & 53 & 14 & 5 & 35.5 & 41 & 11 & 4.5 & 34.5 \\
\hline 61 & 1.5 & 5.5 & 30.5 & 65 & 13.5 & 5 & $3 i$ & 30 & 11 & 3.5 & 31.5 & 77 & 17 & 6.5 & 38 & 38 & 12 & 4 & 33 \\
\hline 58 & 12.5 & 5 & 40 & 47 & 13 & 5 & 38.5 & 42 & 11.5 & 4 & 34.5 & 53 & 13 & 5.5 & 42 & 44 & 13 & 5 & 38.5 \\
\hline 63 & 14 & 6 & 42.5 & 51 & 12 & 5 & 41.5 & 33 & 11.5 & 3.5 & 30.5 & 46 & 12 & 5 & 41.5 & 55 & 13 & b & 38.5 \\
\hline 57 & 13 & 5.5 & 44.5 & 59 & 13.5 & 5.5 & 40.5 & 48 & 13.5 & 5 & 37 & 57 & 12 & 5 & 41.5 & 53 & 13.5 & 5 & 37 \\
\hline 78 & 16.5 & 7 & 42.5 & 73 & 14.5 & 6.5 & 44.5 & 46 & 12.5 & 4.5 & 36 & 51 & 13 & 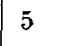 & 38. & 63 & 14 & 5.5 & 38 \\
\hline 59 & 13.5 & 5.5 & 40.5 & 52 & 15 & 5.5 & 30.5 & 66 & 13 & 6 & 46 & 37 & 12 & 4.5 & 36 & 49 & 13 & 4.5 & 39.5 \\
\hline 79 & 17 & 6.5 & 38 & 53 & 12 & 5 & 41.5 & 41 & 11 & 4 & 36.5 & 43 & 13 & 5 & 38.5 & 99 & 12.5 & 4 & 32 \\
\hline 85 & 17 & 7.5 & 44 & 57 & 13 & 5.5 & 44.5 & 41 & 11.5 & 4.5 & 39 & 34 & 10 & 4 & 40 & 37 & 13 & 4 & 30. \\
\hline 92 & 16.5 & 6 & 30.5 & 57 & 15 & 5 & 33 & 35 & 11 & 4 & 36.5 & 50 & 11 & 5 & 45.5 & 45 & 12 & 5 & 41.5 \\
\hline 96 & 17 & 6.5 & 38 & 59 & 14 & 5.5 & 39 & 34 & 11.5 & 4 & 34.5 & 48 & 13 & 5 & 38.5 & 63 & 14 & 6 & 37.5 \\
\hline 62 & 14 & $\mathbf{5}$ & 35.5 & 75 & 15 & 6.5 & 43 & 40 & 11 & 5 & 45.5 & 35 & 12 & 5 & 41.5 & 46 & 11.5 & 5 & 43.5 \\
\hline 73 & 16.5 & 5.5 & 33 & 52 & 11.5 & 4.5 & 39 & 31 & 11 & 4.5 & 41 & 52 & 13 & 0 & 38.5 & 45 & 12 & 5 & 41.5 \\
\hline 85 & 17.5 & 7 & 40 & 52 & 12 & 5 & 41.5 & 36 & 12 & 4.5 & 36 & 32 & 10 & 3.5 & 35 & 50 & 12.5 & 5.5 & 44 \\
\hline 46 & 12 & 5 & 41.5 & 64 & 13.5 & 5.5 & 40.5 & 54 & 13 & 5 & 38.5 & 33 & 11 & 4 & 36.5 & 58 & 12.5 & 5 & 37 \\
\hline 52 & 14 & 5 & $37 . j$ & 50 & 14 & 6 & 42.5 & 69 & 14. & 0 & 41.5 & 38 & 11. & 4 & 34.5 & 57 & 14 & 5.5 & 39 \\
\hline 62 & 13 & 5. & |44.5 & 38 & 11. & 4. & & 55 & 12.5 & b & 40 & 351 & 11 & 4 & |36.5 & 68 & 12.5 & 6 & 44.5 \\
\hline
\end{tabular}


Tabella VII.

\begin{tabular}{|c|c|c|c|c|c|c|c|c|c|c|c|c|c|c|c|c|c|c|c|}
\hline 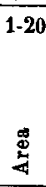 & 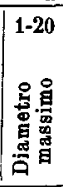 & 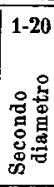 & 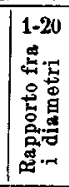 & $\stackrel{\Phi}{\frac{\Phi}{4}}$ & 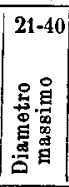 & 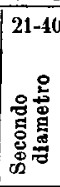 & 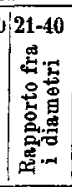 & $\overline{\mid 41-60}$ & 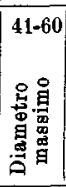 & 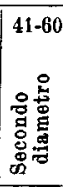 & 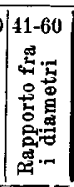 & 趝 & 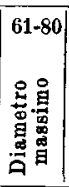 & 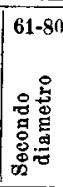 & 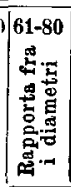 & $\mid 81-100$ & 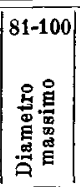 & 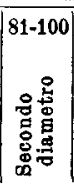 & 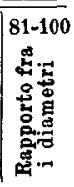 \\
\hline 37 & 8 & 6 & 75 & 36 & 9 & 7 & 78 & 35 & 7.5 & 6.5 & 86.5 & 43 & 9 & 8 & 89 & 38 & 8 & 7 & 87.5 \\
\hline 41 & 8 & 6.5 & 81 & 38 & 7.5 & 7 & 93 & 42 & 9 & 7 & 78 & 48 & 9.5 & 8 & 84 & 38 & 8 & 7 & 87.5 \\
\hline 36 & 8 & 7 & 87.5 & 43 & 8.5 & 7 & 82 & 39 & 8 & 6.5 & 81 & 46 & 8 & 7.5 & 73.5 & 48 & 8.5 & 7.5 & 88 \\
\hline 34 & 7.5 & 6.5 & 86.5 & 51 & 8.5 & 7.5 & 88 & 52 & 9 & 8 & 89 & 49 & 8 & 8 & 100 & 39 & 8.5 & 7 & 82 \\
\hline 44 & 8 & 7 & 87.5 & 44 & 8.5 & 7.5 & 88 & 53 & 9 & 8 & 89 & 45 & 8.5 & 7 & 82 & 40 & 8.5 & 7.5 & 88 \\
\hline 35 & 7.5 & 5.5 & 73 & 38 & 8.5 & 6.5 & 76 & 45 & 8.5 & 8 & 94 & 38 & 8 & 6 & 75 & 40 & 9 & 7 & 78 \\
\hline 32 & 7.5 & 5.5 & 73 & 44 & 9 & 7 & 77.5 & 44 & 9 & 8 & 89 & 44 & 8 & 7 & 87.5 & 49 & 8.5 & 7.5 & 88 \\
\hline 36 & 8 & 6 & 75 & 34 & 7.5 & 7 & 93 & 38 & 9 & 7 & 78 & 53 & 9.5 & 8 & 84 & 43 & 8.5 & 7 & 82 \\
\hline 34 & 7 & 5.5 & 78.5 & 31 & 8 & 6.5 & 81 & 50 & 9.5 & 7.5 & 79 & 28 & 7 & 6 & 85.5 & 41 & 8.5 & 7 & 82 \\
\hline 30 & 7 & 6 & 85.5 & 29 & 7 & 5.5 & 78.5 & 31 & 7.5 & 6 & 79.5 & 35 & 8 & 6 & 75 & 38 & 8 & 6.5 & 81 \\
\hline 40 & 8.5 & 8.5 & 100 & 27 & 7 & 6 & 88.5 & 32 & 7 & 6 & 85.5 & 41 & 8.5 & 7 & 82 & 36 & 8 & 6 & 75 \\
\hline 46 & 9 & 8 & 89 & 30 & 7 & 6 & 85.5 & 35 & 8 & 6 & 75 & 33 & 8 & 6 & 75 & 30 & 8 & 6 & 75 \\
\hline 54 & 9.5 & 9 & 94.5 & 31 & 7.5 & 6 & 79.5 & 42 & 8 & 6.5 & 81 & 37 & 7.5 & 6.5 & 86.5 & 30 & 8 & 7 & 87.5 \\
\hline 50 & 9.5 & 8.5 & 89.5 & 51 & 9.5 & 8 & 84 & 43 & 8 & 7 & 87.5 & 42 & 8 & 6.5 & 81 & 38 & 8.5 & 7 & 82 \\
\hline 51 & 9 & 8 & 89 & 41 & 9 & 8 & 89 & 43 & 7.5 & 7 & 93 & 29 & 7.5 & 6 & 79.5 & 36 & 8 & 7.5 & 93.5 \\
\hline 43 & 9 & 7 & 78 & 28 & 7 & 6 & 85.5 & 50 & 8 & 7.5 & 93.5 & 43 & 9 & 7 & 78 & 44 & 8 & 7 & 87.5 \\
\hline 50 & 9 & 8 & 89 & 36 & 7.5 & 6 & 79.5 & 36 & 7 & 6 & 85.5 & 31 & 7.5 & 6 & 79.5 & 52 & 9 & 8 & 89 \\
\hline 48 & 9.5 & 7.5 & 79 & 36 & 8 & 6 & 75 & 33 & 7 & 6 & 85.5 & 33 & 8 & 7 & 87.5 & 31 & 8 & 7 & 87.5 \\
\hline 49 & 9 & 7.5 & 83.5 & 37 & 8.5 & 7 & 82 & 37 & 7.5 & 6.5 & 86.5 & 41 & 8.5 & 7 & 82 & 52 & 9.5 & 8 & 84 \\
\hline 56 & 9.5 & 8 & 84 & 55 & 9 & 9 & 100 & 42 & 8 & 7 & 87.5 & 33 & 8 & 7 & 87.5 & 40 & \begin{tabular}{|l}
9.5 \\
\end{tabular} & 8 & 84 \\
\hline
\end{tabular}

Da queste tabelle rileviamo che l'aree delle cellule di rivestimento e di quelle glandolari non offrono grandi differenze, oscillando ad un dipresso fra gli stessi estremi: pure questi estremi sono meno ampi per le cellule glandolari che per quelle di rivestimento. I rapporti invece fra $i$ due diametri massimi, mostrano dei dati molto diversi. Nelle cellule glandolari essi variano da 50 a 100; i rapporti più frequenti sono 70 e quelli fra 86 e $92: 42$ su 100 cellule mostrano tale rapporto.

Nelle cellule di rivestimento, al contrario, esso oscilla da 20 a 38 ; per 71 di 100 nuclei questo rapporto varia tra 22 e 28.

Dati analoghi ei sono forniti dai nuclei delle cellule stesse.

Quanto all' area notiamo che essa è maggiore nei nuclei delle cellule di rivestimento che in quelli delle cellule glandolari. Il rapporto fra i diametri oscilla per i nuclei di rivestimento tra 30 e 46, raggiungendo il massimo tra 36 e 42, essendo tale il rapporto di 72 su 100 nuclei. Nei nuclei delle cellule glandolari esso oscilla tra 74 e 100. 
Ricerche sulla differenziazione cellulare nello svilappo embrionale.

Fig.e.

Fig. $c$.
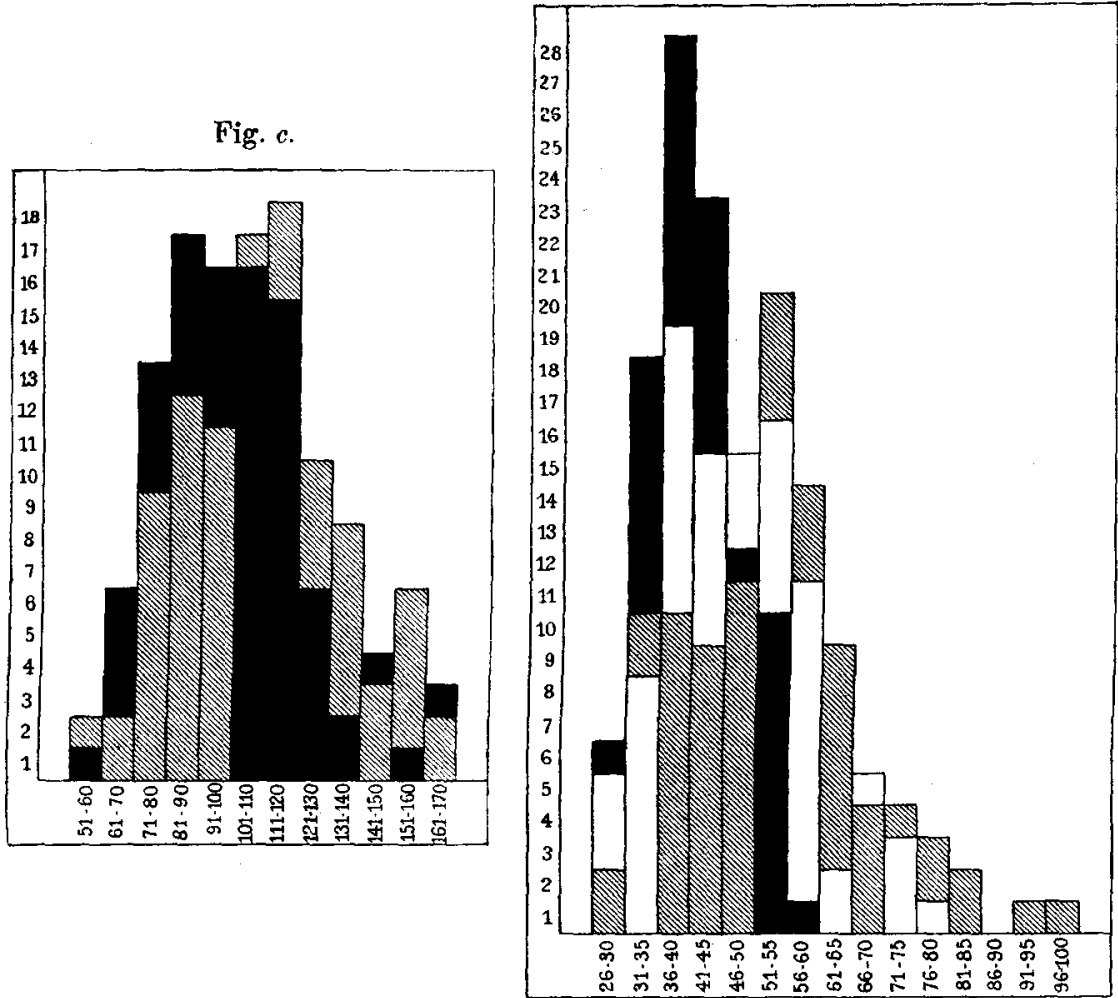

Fig. $d$.

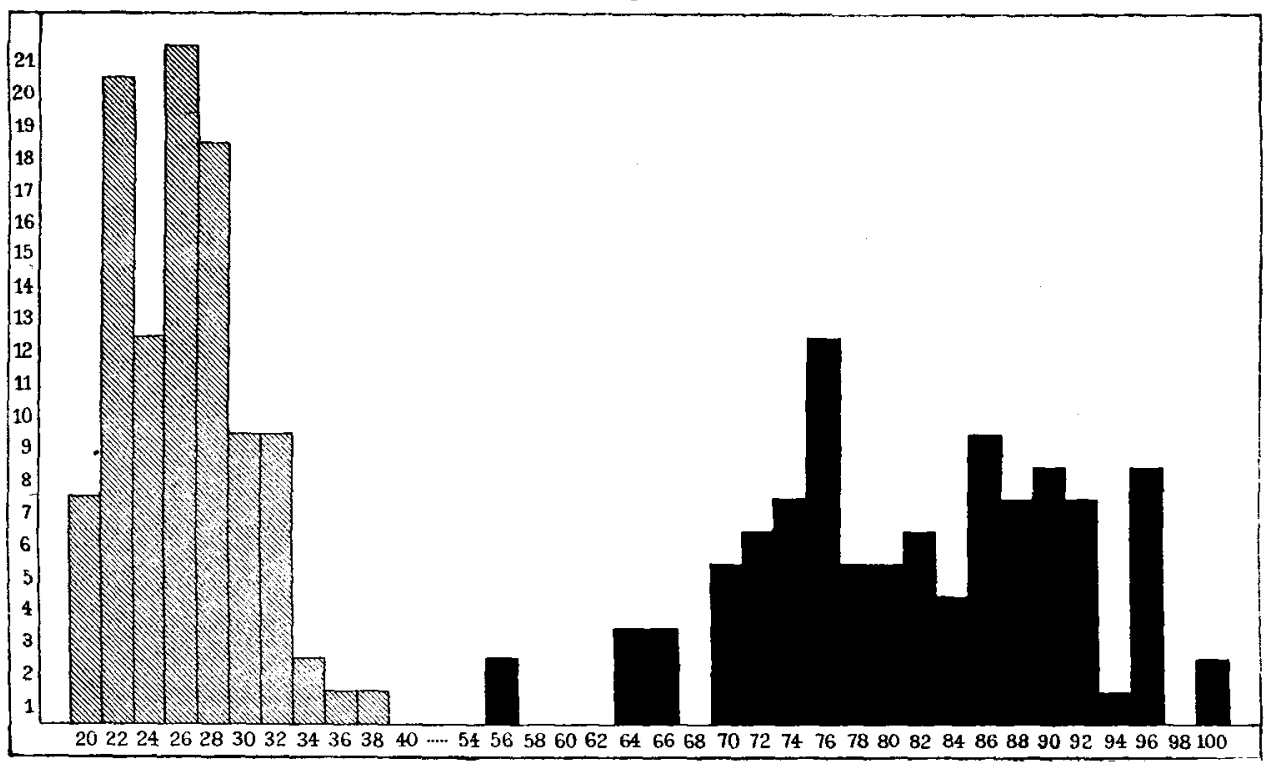


Auche qui raccogliamo in diagrammi i dati ricavati dalle misurazioni.

Il diagramma della fig. $c$ rappresenta la curva delle aree nelle due specie di cellule; la fig. $d$ quella dei rapporti fra i diametri massimi delle cellule stesse. La fig. $e$ poi ci dà l'area e la fig. $f$ i rapporti fra i diametri dei nuclei di queste cellule.

Le aste chiare rappresentano le cellule ed i nuclei di rivestimento, quelle più scure le cellule glandolari ed i nuclei loro. Anche

Fig. $f$.

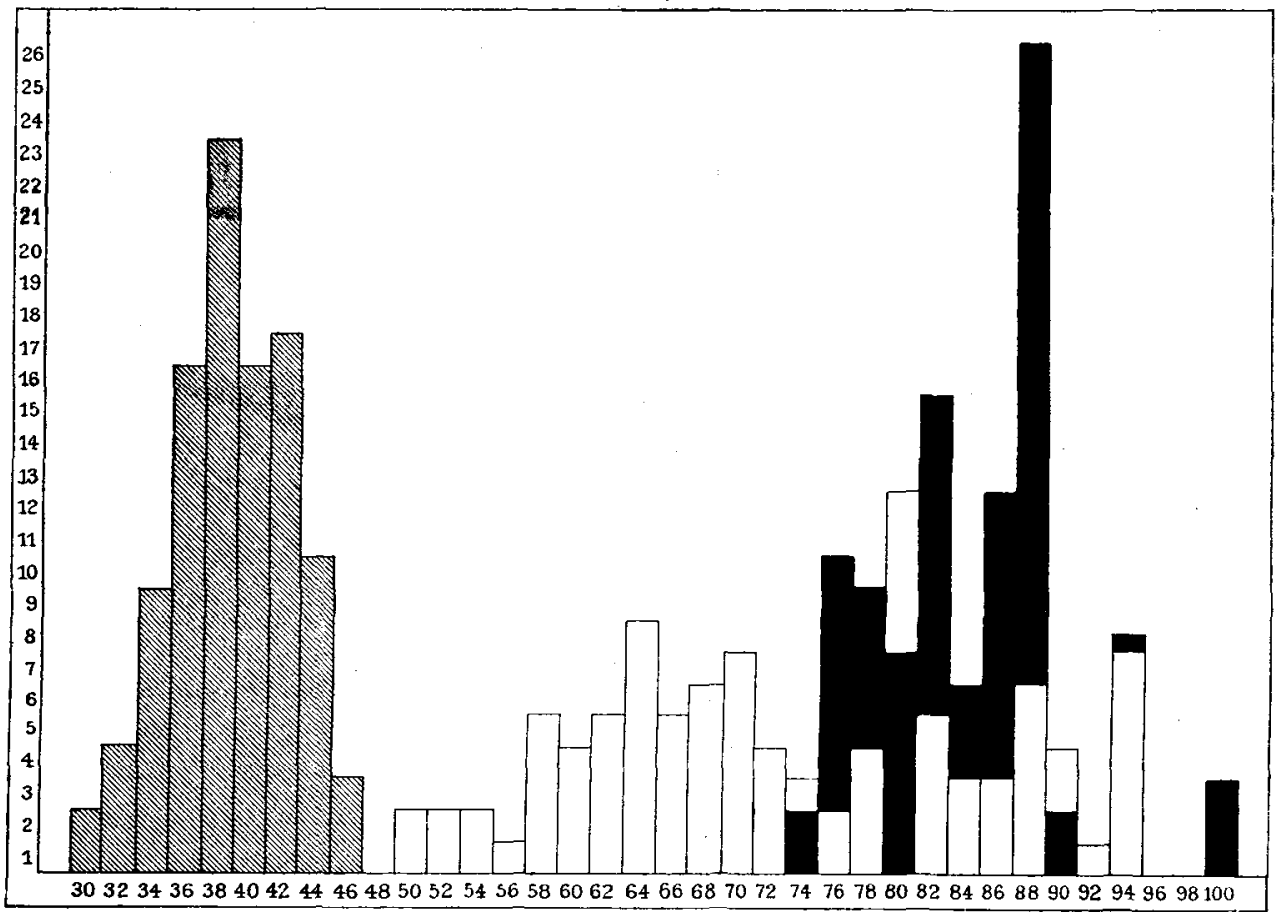

qui jper facilità del confronto riportiamo nella fig. $f$ la curva dei nuclei non ancora differenziati; essa è rappresentata dalle aste in bianco.

Quanto alle altre indicazioni rimandiamo alla spiegazione della fig. $a$ e rispettivamente della fig. $b$.

L'osservazione del diagramma della fig. $f$ ci rivela alcuni dati molto importanti: la eurva dei nuclei di rivestimento oceupa un tratto dell' ascissa del tutto distinto da quello, su cui si eleva quella dei naclei glandolari: quella poi dei nuclei indifferenziati occupa una posizione intermedia fra le due. 
Ma uno speciale interesse può avere il confronto di questo diagramma con quello della fig. $b$, tanto più se teniamo presente la struttura istologica dei rispettivi elementi.

La curva dei rapporti fra i diametri dei nuclei di rivestimento non è molto dissimile da quella relativa ai nuclei riprodotti nella fig. 2; essa si estende però fra due limiti inferiori a quelli di quest' ultima: la forma allungata è più accentuata nei nuclei di rivestimento che in quelli prima descritti.

Al contrario, i nuclei delle cellule glandolari mostrano una curva, che rassomiglia a quella dei nuclei rappresentati nella fig. 3, raggiungendo tutte e due allo stesso punto il loro fastigio. Gli estremi della prima, però, sono più ravvicinati fra loro, che quelli della seconda: il che vuol dire che in complesso i nuclei glandolari tendono ancor più ad assumere la forma circolare, che non i nuclei rotondeggianti del secondo stadio di differenziazione.

Dal confronto poi della struttura morfologica di questi elementi, possiamo ritenere che $\mathrm{i}$ nuclei di rivestimento sieno un grado più avanzato di- differenziazione, dei nuclei allungati dello stadio precendente di sviluppo; quelli glandolari invece deriverebbero dai nuclei rotondeggianti dello stadio stesso.

Potremo perciò dire, che i rapporti geometrici dei nuclei vanno differenziandosi, da un' origine comune, in due direzioni opposte, raggiungendo il massimo della differenziazione nei nuclei di rivestimento, da una parte, ed in quelli glandolari, dall' altra, passando per stadi intermedi, rappresentati dai nuclei già prima descritti. Questa differenziazione, che chiamęò geometrica, procede di pari passo eon quella istologica dei nuclei stessi.

Quanto poi alle cellule glandolari ed a quelle di rivestimento, troviamo ripetuti, per quello che concerne $i$ rapporti dei diametri, $i$ fatti che si riscontrarono nei rispettivi nuclei, essi sono però esagerati nelle cellule di rivestimento, perchè il citoplasma è, quasi tutto, disposto nella direzione del diametro maggiore del nucleo e manca quasi nella direzione dell' altro diametro.

Le misurazioni di queste cellule ripetute nei vari giorni di sviluppo, hanno sempre dato gli stessi risultati.

Cosi pure le misurazioni di tutti gli altri elementi, fatte per ciascuno stadio di differenziazione ripetutamente nei vari giorni di sviluppo, hanno sempre dato cifre costanti.

Abbiamo già più su avvertito, che la differenziazione degli elementi comincia dall' estremo orale dell' intestino, e si estende da 
questo verso l'estremità opposta. I varii tratti dell' intestino si trovano perciò contemporaneamente a diversi gradi di sviluppo; difatti, mentre in

Fig. g.

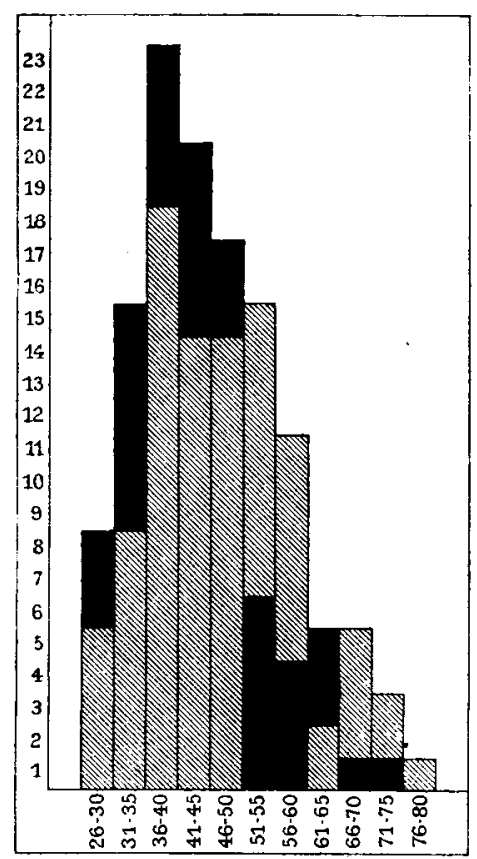
un primo segmento dell' intestino in un embrione per es. di 26 o 28 giorni si trovano già sviluppate le cellule di rivestimento e glandolari, in un altro tratto non si riscontrano ancora, che elementi che sono allo stadio descritto come secondo.. In un terzo segmento ancora vediamo i nuclei ed il citoplasma per nulla ancora differenziati, appunto quali si trovano nell' intestino di embrioni ai primi giorni di sviluppo, quando ancora non è formato il lume intestinale. Tale mancanza di differenziazione si riscontra ancora negli embrioni appena sgusciati dal mueo.

Tutte le dimensioni sono in questi nuclei eguali a quelle trovate per i primi giomi di sviluppo, come si nota nei diagrammi $g$ ed $h$, di cui il primo ei dà l'area ed il secondo il rapporto fra $\mathrm{i}$ diametri nei nuclei indifferenziati di un embrione di 13 giorni, ed in quelli dell ultima parte dell' intestino di uno di trenta giorni.

Come si vede da queste curve non esiste alcuna differenza fra i due ordini di nuclei, se non quelle dovute alle differenze individuali

Fig. $h$.

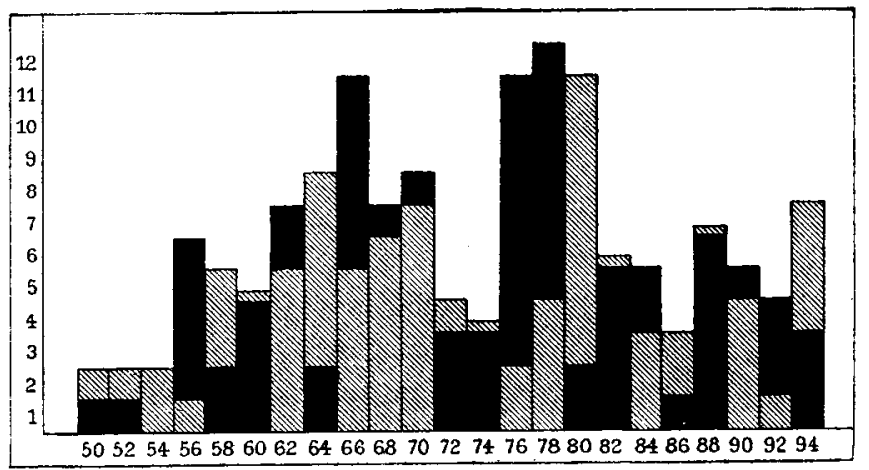


esistenti fra nucleo e nucleo, ed all' imperfezione del metodo, consistente principalmente nel fatto, che non tutti $\mathrm{i}$ nuclei vengono sezionati (e quindi misurati) nel loro piano maggiore, ma in uno, a questo più 0 meno inferiore.

Le aste chiare si riferiscono ai nuclei del tredicesimo giorno di sviluppo, quelle più seure a quelli del trentesimo giorno.

Non occorre avvertire che l'indicazioni, che si riferiscono ai giorni di sviluppo, sono del tutto relative e che qua si riferiscono al tempo occupato dai miei embrioni per il loro sviluppo, tempo che può variare molto a seconda della temperatura e di altre cause.

Ricapitoliamo brevemente quanto abbiamo fino a qui esposto.

Negli embrioni di tritone osserviamo, che gli elementi dell'intestino si mantengono invariati per un tempo più o meno lungo, nei diversi tratti dell' organo stesso.

La differenziazione istologica che incomincia dall' estremo orale dell' intestino, è accompagnata da una variazione limitata della grandezza dell' area degli elementi, e da una variazione molto considerevole dei rapporti fra i diametri degli elementi stessi, variazione, che si estende perciò altresi al loro volume e specialmente al rapporto fra le varie parti della massa dell' elemento e la sua superficie.

Questa variazione aumenta quanto più progredisce la differenziazione istologica, e nelle due specie di quest' ultima procede in due direzioni diverse.

Le dimensioni invece ed i rapporti geometrici di ciascun elemento, per ogni stadio di differenziazione, sono eguali qualunque sia l'età dell' embrione.

Oltre a questo parallelismo fra struttura geometrica e differenziazione istologica, è degno di nota anche il modo, in cui il dentoplasma si comporta rispetto alla differenziazione istologica stessa.

Possiamo riassumere le osservazioni su quest' argomento dicendo, che non si avvera nessun mutamento nella struttura degli elementi intestinali, sino a che il deutoplasma vi si trova in grande abbondanza; quando invece questo comincia a diminuire, s'inizia la differenziazione istologica. Questa e la diminuzione del deutoplasma procedono poi di pari passo.

Di più è da notare, che negli elementi, iu cui è più sviluppata 
una delle due direzioni del piano di sezione, i granuli di deutoplasma si dispongono di preferenza (non esclusivamente) nella direzione della dimensione maggiore, e ciò tanto più, quanto più grande è la differenza fra $i$ due diametri (vedi fig. 2, 4, ed 5). Manca invece una localizzazione riconoscibile del deutoplasma, quando poca è la diversità fra $\mathrm{i}$ due diametri.

Riassumendo tutto ciò, il fatto più notevole e significante.di queste osservazioni, si $\dot{e}$, che la differenziazione istologica degli elementi, va di pari passo, da un lato colla modificazione dei loro rapporti geometrici, dall' altro col diminuire e col localizzarsi della sostanza nutritiva immagazzinata nell' novo, del deutoplasma.

Quale sarà l'interpretazione fisiologica che potremo dare di questi fatti?

II".

La struttura geometrica degli elementi intestinali in embrioni ottenuti da uno solo dei due primi blastomeri.

Esaminato nel capitolo precedente quanto durante la differenziazione cellulare si osserva nell intestino di embrioni normali di tritoni, sarà opportuno, prima di tentare una risposta alla dimanda, che alla fine del capitolo stesso ci siamo rivolti, cercare un controllo dei risultati ottenuti, su un materiale di studio diverso.

A questo scopo, ho creduto, potere utilizzare con vantaggio gli embrioni, ottenuti da mezzo uovo solo di tritone.

Riferirò qui brevemente quanto altra volta ho avuto occasione di osservare su questi embrioni, per quanto ciò possa concernere l'argomento di queste ricerche. Ho potuto allora vedere, che in questi embrioni, mentre il numero delle cellule per ciascuna sezione trasversale era in alcuni organi normale ed in altri ridotto alla metà, la grandezza invece degli elementi, calcolata sul loro diametro massimo, era normale, tanto negli organi forniti del numero normale di cellule - per sezione trasversale - quanto in quelli, in cui tale numero era ridotto a metà.

Tale misurazione non era però stata eseguita, per gli elementi intestinali, nè il metodo usato allora, la misurazione cioẻ del diametro massimo, corrisponde al metodo della attuale ricerca, in cui oltre che conoscere la grandezza degli elementi, ci interessa studiarne la forma, che ci viene rilevata dal rapporto dei due diametri massimi. 
Per vedere quindi, se i fatti riscontrati nell' embrione normale, trovavano la loro conferma, in quanto arviene negli embrioni che si sono sviluppati da un solo dei primi blastomeri, ho ripreso lo studio dei preparati di questi embrioni, ed ho sui loro elementi ripetute le misurazioni già eseguite nell embrione normale.

Pur troppo però questi embrioni, che non erano destinati a servire per studi citologici, erano stati colorati con sostanze che poco si prestano a mettere in evidenza il citoplasma, come è specialmente l'emallume di MEYER, che è un colore quasi esclusivamente nucleare. Se in altri organi il limite cellulare, molto evidente per sè, si può riconoscere molto bene anche con questa colorazione, nell' intestino invece, il confine fra cellula e cellula ha bisogno per essere distinto, di esser messo in evidenza dalla colorazione citoplasmatica. Percio ho dovuto rinunziare alla misurazione delle dimensioni delle cellule in queste embrioni, se non ho voluto espormi al pericolo di incorrere in errori molto grossolani.

E' quindi sui nuclei di questi embrioni che ho eseguito il controllo dei risultati del capitolo precedente.

Ho eseguite le misurazioni sui nuclei dei vari tratti dell' intestino di questi embrioni, appena sgusciati dal muco. Nel tratto più orale si hanno i nuclei più differenziati, quelli di rivestimento e quelli glandolari, in un secondo tratto troviamo un grado di differenziazione minore, corrispondente alle figure 2 e 3 ; più verso l'ano ancora l'intestino è perfettamente indifferenziato.

Le misurazioni eseguite su questi nuclei hanno dimostrato, che in tutti gli stadi ed in tutte le forme di differenziazione, la grandezza dell' area ed il rapporto fra i diametri massimi del nucleo, sono eguali negli embrioni normali ed in quelli ottenuti da un solo dei due primi blastomeri. Soltanto l'area dei nuclei indifferenziati si mostra complessivamente un poco maggiore in quest' ultimi, e ciò conferma quanto dissi più sopra, che $\mathrm{i}$ valori più bassi per l'area dei nuclei negli embrioni normali, dipendeva dal fatto che la sezione e quindi la misurazione non avveniva sempre in corrispondenza dello spessore massimo del nucleo, per la sottigliezza della sezioni.

Di fatti in questi embrioni le sezioni erano più grosse $\left(\begin{array}{llll}7 & \text { o } & 8 & \mu\end{array}\right)$ sicehè ciascun nucleo non occupava all' incirca che una sezione sola, e poteva venire quindi misurato nella sua massima grandezza. I nuclei di rivestimento invece, per il loro piccolo spessore trasversale, occupano anche nelle sezioni sottili una sola di queste; nell' area di tali nuclei non si nota perciò nessuna diversità nelle due specie 
di embrioni. Perciò la piccola diversità fra le aree dei nuclei indifferenziati degli embrioni normali e di quelli sperimentali, ehe si nota nelle seguenti tabelle è più apparente che reale.

Dei dati raccolti dalle nostre misurazioni, riportiamo qui solo due tabelle, essendo inutile riempire di più queste pagini di tante cifre, giacchè le tabelle che seguono bastano a provare quanto abbiamo più su asserito. Esse si riferiscono ai due estremi della differenziazione; la tabella VIII ci dà le dimensioni dei nuclei affatto indifferenziati, negli embrioni di controllo, la IX i nuclei delle cellule di rivestimento dell' intestino negli stessi embrioni. Le tabelle che si riferiscono alle altre differenziazioni, e che qua ommettiamo, offrono dati analoghi, corrispondenti cioè, a quelle delle rispettive differenziazioni negli embrioni normali.

Tabella VIII.

\begin{tabular}{|c|c|c|c|c|c|c|c|c|c|c|c|c|c|c|c|c|c|c|c|}
\hline 离 & 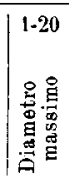 & 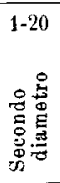 & 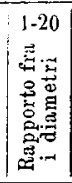 & $21-10$ & 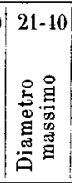 & 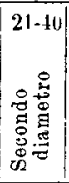 & 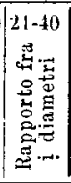 & $|+1-60|$ & 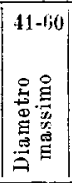 & 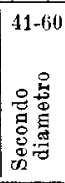 & 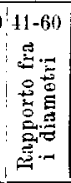 & $|61-50|$ & 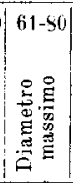 & 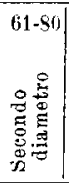 & 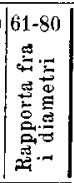 & $|81-1013|$ & 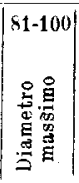 & 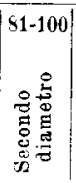 & 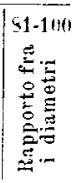 \\
\hline 49 & 9 & 8 & 89 & 56 & 10.5 & 7.5 & 71.5 & 49 & 10 & 6.5 & 65 & 47 & 10 & 6.5 & 65 & 43 & 8.5 & 6.5 & 71 \\
\hline 42 & 8.5 & 7 & 82 & 42 & 9 & 6 & 66.5 & 34 & 8.5 & 6 & 70.5 & 45 & 9 & 6.5 & 172 & 45 & 8.5 & 6.5 & 71 \\
\hline 48 & 9.5 & 7 & 73.5 & 39 & 8 & 5 & $6 \div .5$ & 37 & 8.5 & 6.5 & 71 & 30 & 8 & 5.5 & 68.5 & 44 & 9 & 7 & 78 \\
\hline 57 & 10 & 8 & 80 & 44 & 9.5 & 6 & 63 & 39 & 9 & 5.5 & 61 & 49 & 10.5 & 6 & 57 & 41 & 10.5 & 6 & $5 \%$ \\
\hline 56 & 10.5 & 7 & 66.5 & 48 & 9 & 8 & 89 & 44 & 8.5 & 6 & 70.5 & 35 & 9.5 & 5 & 52 & .38 & 8 & 5.5 & 68.j \\
\hline 36 & 8.5 & 6 & 70.5 & 50 & 10 & 7 & 70 & 36 & 9 & 5 & 55.5 & 44 & 9 & 7 & 78 & 37 & 9.5 & 5.5 & 58 \\
\hline 42 & 10 & 7 & 70 & 39 & 8.5 & 6 & $70 . \check{3}$ & .33 & 7 & 5.5 & 78.5 & 37 & 9 & 6 & 66.5 & 36 & 9 & 5 & 55.5 \\
\hline $3 \tilde{5}$ & 8.5 & 6.5 & 86.5 & 39 & 9 & $\overline{5} . \overline{3}$ & 61 & 45 & 8. & 7 & 82 & 46 & 9 & $7 . \tilde{5}$ & 83.5 & 58 & 9 & 8.5 & 94.5 \\
\hline 43 & 9.5 & 7 & 73.5 & 57 & 10.5 & 6.5 & 62 & 43 & 9.5 & 6 & 63 & 36 & 9 & $\check{j}$ & $5 \overline{5} .5$ & 43 & 10.5 & 6 & 57 \\
\hline 49 & 9.5 & 7 & 73.5 & 48 & 10 & 6 & 60 & 37 & 9 & 5.5 & 61 & 52 & 9 & 8 & 89 & 60 & 11.5 & 7.5 & 65.5 \\
\hline 68 & 10.5 & 8.5 & 81 & 66 & 10 & $8 . \overline{0}$ & 85 & 39 & 8.5 & 5.5 & 64.5 & 43 & 10 & 6 & 60 & 45 & 11. & 5.5 & 50 \\
\hline 57 & 10 & 7.5 & 75 & 50 & 9.5 & 7 & 73.5 & 26 & 9 & 4.5 & 50 & 43 & 11 & 6 & 54.5 & 69 & 10.5 & 9.5 & 90.5 \\
\hline 48 & 8.5 & 7 & 82 & 34 & 9 & 5.5 & 61 & 57 & 11.5 & 7.5 & 65.5 & 50 & 10.5 & 7.5 & 71.5 & 54 & 11 & 6 & 54.5 \\
\hline 49 & 9.5 & 8 & 81 & 47 & 9.5 & 5.5 & 58 & 44 & 10 & 5.5 & $5 j$ & 34 & 9 & 6 & 66.5 & 56 & 10 & 7 & 70 \\
\hline 38 & 7.5 & 6 & 80 & 33 & 9.5 & 5 & ว2.5 & 45 & 9 & $r$ & 78 & 38 & 8.5 & 6 & 70.5 & 68 & 11 & $8 . \overline{5}$ & 77 \\
\hline 54 & 11 & 7.5 & 68 & 48 & 10.5 & 6.5 & 62 & 40 & 9 & 5.5 & 61 & 43 & 9.5 & 6.5 & 68.5 & 63 & 10 & 9 & 90 \\
\hline 58 & 10.5 & 8 & 76 & 59 & 10.5 & 7.5 & 71.5 & 49 & 9 & 7.5 & 83.5 & 32 & 7 & 5 & 78.5 & 45 & 10 & 6.5 & 65 \\
\hline 47 & 5 & 7.5 & 93.5 & 38 & 7.5 & 7 & 43 & 33 & $8 . \check{5}$ & 5 & 58.5 & 40 & 10.5 & 6 & 57 & 43 & 9.5 & 6.5 & 68.5 \\
\hline 42 & 7.5 & 7 & 93 & 68 & 10.5 & 8 & 76 & 45 & 9 & 6.5 & 72 & 52 & 10 & 6.5 & 65 & 53 & & 8 & 89 \\
\hline 44 & 10 & 6 & 60 & 44 & 9 & 6 & 60.5 & 52 & 10.5 & 6 & 57 & 48 & 8 & 7 & 87.5 & 50 & 9.5 & 8 & 84 \\
\hline
\end{tabular}


Tabella IX.

\begin{tabular}{|c|c|c|c|c|c|c|c|c|c|c|c|c|c|c|c|c|c|c|c|}
\hline$\stackrel{8}{\square}$ & 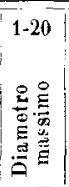 & 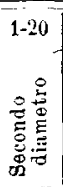 & 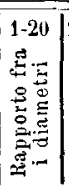 & $|21-40|$ & 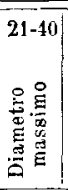 & 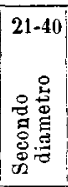 & 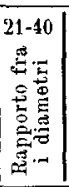 & 㟢 & 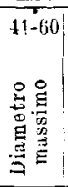 & 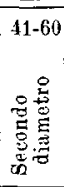 & 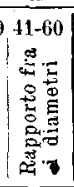 & 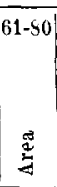 & 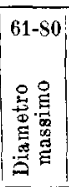 & 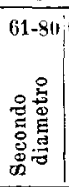 & 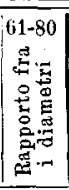 & $|81-100|$ & 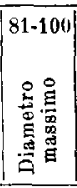 & 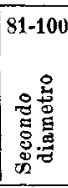 & 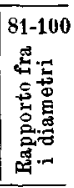 \\
\hline 56 & 14 & 6 & 42.5 & 58 & 12.5 & 5 & 40 & $4 i$ & 13 & 5 & 38.5 & 58 & 14 & 5.5 & 39 & 43 & 11.5 & 4.5 & 37.5 \\
\hline 78 & 14.5 & 6.5 & 44.5 & 39 & 11.5 & 5 & 43 & 46 & 12.5 & 5 & 40 & 70 & 14.5 & 5.5 & 38 & 73 & 14.5 & 5.5 & 38 \\
\hline 58 & 12.5 & 5 & 40 & 37 & 11 & 4.5 & 40 & 51 & 13 & 6 & 16 & 75 & 14 & 6 & 41.5 & 55 & 13.5 & 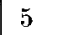 & 37 \\
\hline 73 & 13.5 & 6.5 & 48 & 44 & 11.5 & 4.5 & 39 & 40 & 12.5 & 5 & 40 & 54 & 13 & 5.5 & 42 & 61 & 13.5 & 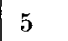 & 37 \\
\hline 43 & 11.5 & 5 & 43.5 & 33 & 11 & 4.5 & 40 & 38 & 11.5 & 4 & 34.5 & 68 & 14 & 6 & 41.5 & 46 & 12 & 4.5 & 37.5 \\
\hline 45 & 12 & 5 & 41.5 & 50 & 13 & 5 & 38 & 52 & 12 & 5.5 & 45 & 38 & 10.5 & 4.5 & 42.5 & 52 & 15 & & 33 \\
\hline 43 & 11 & 4.5 & 41 & 38 & 11.5 & 4.5 & 39 & 41 & 12 & 5 & 41.5 & 45 & 13 & 5 & 38.5 & 52 & 13 & 5.5 & 42 \\
\hline 47 & 12.5 & 5 & 40 & 47 & 12 & 5 & 41 & 38 & 11.5 & 4 & 34 & 44 & 12 & 4.5 & 37.5 & 26 & 11.5 & & 34.5 \\
\hline 50 & 12.5 & 4.5 & 36 & 41 & 10.5 & 4.5 & 42.5 & 46 & 11 & 5 & 45.5 & 46 & 11 & 5 & 45.5 & 46 & 13 & & 38.5 \\
\hline 49 & 13 & 5.5 & 42 & 51 & 11.5 & 5 & 43 & 40 & 12.5 & 4.5 & 36 & 53 & 13 & 5 & 38.5 & 58 & 13.5 & 5.5 & 40.5 \\
\hline 57 & 13 & 5.5 & 42 & 44 & 11 & 4.5 & 40 & 37 & 11 & 3.5 & 31.5 & 39 & 12 & 4.5 & $3 \tau .5$ & 50 & 14 & 5.5 & 39 \\
\hline 56 & 12.5 & 5.5 & 44 & 42 & 10 & 4.5 & 45 & 30 & 10 & 4 & 40 & 30 & 10 & 3.5 & 35 & 58 & 14.5 & 5.5 & 36.5 \\
\hline 59 & 13 & $\tilde{5} . \tilde{3}$ & 42 & 35 & il & 3.5 & 31 & 36 & 10 & 4.5 & 45 & 78 & 14.5 & 6 & 41.5 & 76 & 15.5 & 6.5 & 42 \\
\hline 46 & 11. & 4.5 & 41 & $5 i$ & 14 & 6 & 42 & 45 & 12.5 & 4.5 & 36 & 47 & 12.5 & 4.5 & 36 & 70 & 15 & 6 & 46 \\
\hline 41 & 10 & 4.5 & 15 & 71 & 13 & 6 & $4 h$ & 45 & 11.5 & 4.5 & 37.5 & 57 & 14 & 5.5 & 38 & 49 & 1 & & 35.5 \\
\hline 39 & 11.5 & 4 & 39 & 56 & $14^{\circ}$ & 6 & 42 & 60 & 13.5 & 5.5 & 40.5 & 53 & 13 & 5.5 & 12 & 38 & 13 & 4.5 & 34.5 \\
\hline 32 & 10 & 3.5 & 35 & 35 & 11.5 & 4.5 & 39 & 28 & 10 & 4 & 10 & 67 & 14.5 & 6 & 41.: & 59 & 14.5 & 5.5 & 36 \\
\hline 45 & 12.5 & 5 & 40 & 39 & 11 & 4.5 & 40 & 30 & 10.5 & 3.5 & 33.5 & 59 & 13 & 5.5 & 42 & 40 & 14 & 4 & 38.5 \\
\hline 50 & 12.5 & 5 & 40 & 40 & 11.5 & 4.5 & 39 & 31 & 10.5 & 4 & 38 & 53 & 12.5 & 5 & 40 & 38 & 11 & 4.5 & 41 \\
\hline 39 & 12.5 & 4.5 & 36 & 48 & 13.5 & 5 & 37 & 34 & 11 & 5 & 45.5 & 57 & 13.5 & 5.5 & 37 & 48 & 13.5 & 5 & 37 \\
\hline
\end{tabular}

Il fatto, ehe da queste tabelle, o ancor meglio dai diagrammi seguenti, appare, che cioè, negli embrioni ottenuti da mezzo novo, aree e rapporti geometrici, sono identici a quelli degli embrioni sviluppati da un uovo intero, prova l'importanza, che per la vita della cellula e del nucleo hanno queste dimensioni: esso infatti dimostra, che, anche quando è ridotta a metà la quantità di materiale da cui trae origine un organismo, gli elementi di questo si riducono di numero, ma non possono cambiare il volume e la forma tipica di ciascuno stadio di differenziazione: sicchè appare doversi ritenere, che alla natura di ciascun elemento vadano legati una data forma ed un dato volume, e che questi ultimi siano indispensabili e costanti per ogni specie di nucleo e di cellula.

I diagrammi delle fig. $i$ e $l$ si riferiscono ai nuclei indifferenziati, degli embrioni ottenuti da mezz' uovo, messi a confronto con quelli dell' embrione normale; i primi sono indicati dalle aste più 
Fig. $i$.

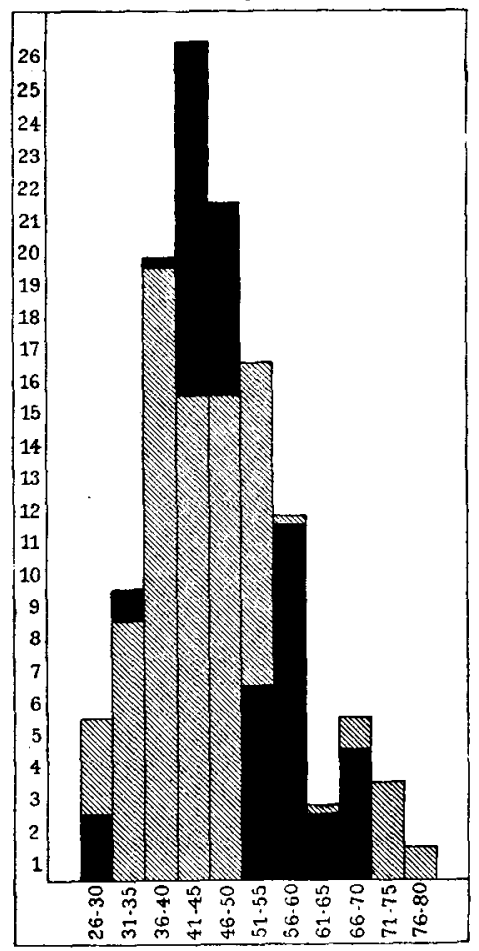

Fig. $m$.

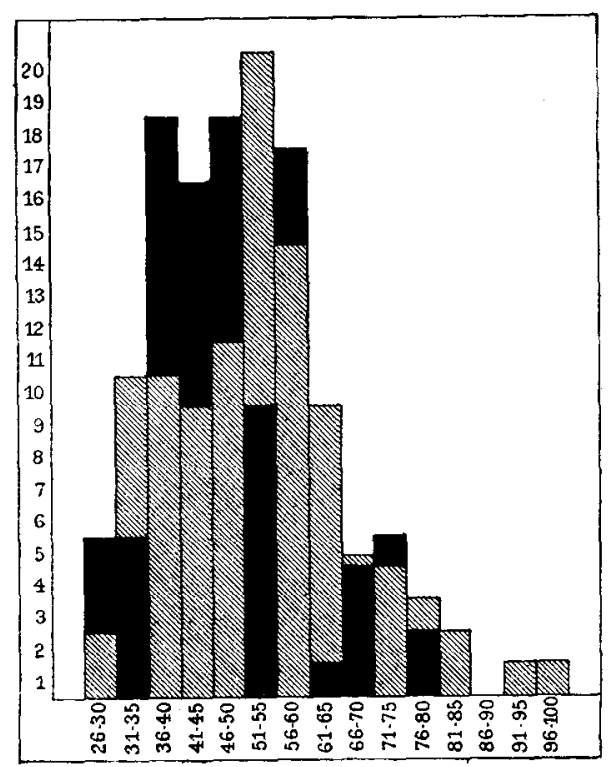

Fig. $l$.

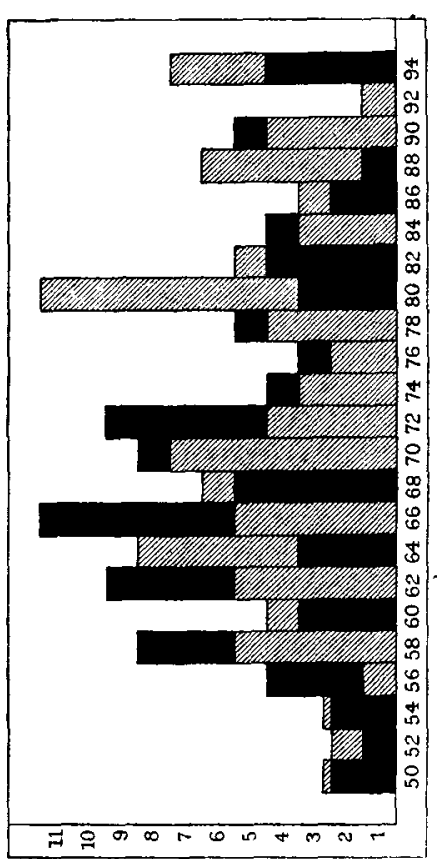

Fig. $n \cdot \vec{i}$

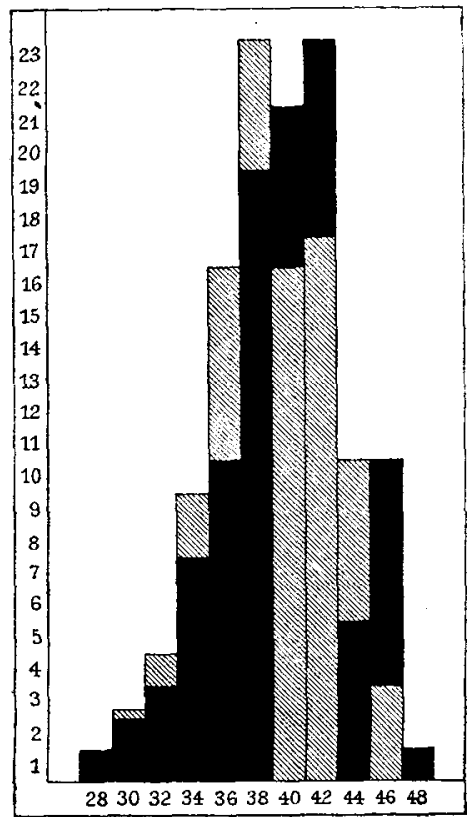


scure' i secondi da quelle chiare; la fig. $i$ ci dà l'area, la fig. $l$ i rapporti di questi nuclei. Analoghe sono le figg. $m$ e $n$ che appartengono ai nuclei di rivestimento.

\section{III.}

\section{Discussione dei risultati e conclusioni.}

Riepiloghiamo brevemente i fatti che siamo andati esponendo nelle pagine precedenti.

$1^{\circ}$ Fino a che il deutoplasma si trova in abbondanza raccolto nelle pareti dell' intestino, gli elementi di questo non subiscono nessuna modificazione, e non si riconoscono limiti fra cellula e cellula.

$2^{\circ}$ Quando comincia a diminuire il deutoplasma, si inizia la differenziazione istologica e geometrica degli elementi, e, solo quando esso è molto diminuito, si riconoscono dei limiti cellulari.

$3^{\circ}$ In ognuna delle forme di differenziazione istologica, qualunque sia l'età dell' embrione, gli elementi hanno eguali i diametri, quindi eguale è il. loro volume, la posizione di ciascuna parte della massa rispetto alla superficie ecc. Ciascuna forma di differenziazione si distingue al contrario dalle altre, oltre che per la struttura istologica anche, in un grado vario, per la sua forma geometrica.

$4^{0}$ Tra la forma geometrica degli elementi più differenziati e quella degli elementi affatto indifferenziati esistono dei gradi intermedi, che corrispondono appunto, a stadi intermedi di differenziazione istologica.

$5^{\circ}$ La forma geometrica per ciascuna differenziazione istologica è costante, non solo negli embrioni normali, ma anche in quelli ottenuti da un solo blastomero.

Da questi fatti appare che la differenziazione istologica è legata alla diminuzione del dentoplasma ed è accompagnata da una modificazione della forma geometrica: questa poi sembra costante per ciascuna specie di differenziazione.

Come possiamo noi spiegarci questi fatti? Quale è il significato che per la cellula, hanno il suo volume ed i suoi rapporti geometrici? Esiste, e se esiste quale è il rapporto fra il dileguarsi del deutoplasma da una parte ed il mutarsi della forma geometrica ed il delimitarsi delle cellule tra loro dall' altra?

E'nota da lungo tempo la funzione del dentoplasma. Esso è una sostanza nutritiva, immagazzinata nell' uovo, e serve allo sviluppo embrionale del nuovo organismo. La scomparsa del deuto- 
plasma è stata seguita da GALEOTTI. I granuli, specie in corrispondenza di cordoni citoplasmatici, si dividono in frammenti sempre piu piccoli che poi vanno a poco a poco scomparendo. Essi vengono cosi digeriti dal citoplasma. Quanto più questi granuli deutoplasmatici vanno scomparendo, aumentano nell' interno del citoplasma i granuli cosidetti fuesinofili o granuli d'escrezione, che GaLEoTTI ed a ragione - ritiene come i prodotti regressivi del metabolismo del protoplasma.

La digestione del dentoplasma avviene quindi nel citoplasma stesso, fra le sue maglie: questa digestione corrisponde a quella endocellulare di alcuni protisti ignudi (amebe ecc.) e dei lencociti durante la fagocitosi. Anche in questi organismi il citoplasma inghiottisce - se è permessa questa parola - delle sostanze nutritive solide e semisolide, ed i prodotti della digestione, che ne avviene, si vanno formando già nell' interno della cellula.

In ambedue i casi perciò, nella digestione del deutoplasma ed in quella endocellulare, il citoplasma non ha bisogno di assorbire dall' esterno la sostanza nutritiva: questa si trova invece fra le sue maglie ed al citoplasma non resta che trasformarla in sostanza vivente. Il solo elemento che il eitoplasma abbia bisogno di assorbire dall'ambiente esterno è l'ossigeno, vale a dire un elemento allo stato gassoso, facilmente diffondibile.

Non è cosi per il nucleo. In questo non ci sono sostanze nutritive, esso per il suo metabolismo ha bisogno di assorbirle allo stato liquido, ed esse devono essere di una grandezza molecolare non troppo grande, per poter essere diffondibili attraverso la membrana nucleare; vale a dire, che per l'assorbimento di una sostanza albuminoide questa dovrà aver subito un processo di idratazione, cioè essere peptonizzata.

Ma il deutoplasma comincia a diminuire ed a localizzarsi in determinate parti del citoplasma. Le parti in cui il deutoplasma è incluso, avranno perciò quelle stesse funzioni digestive di cui godevano prima: queste funzioni invece verranno a mancare nelle parti sfornite di deutoplasma: esse dovranno perciò ricevere le sostanze nutritive, o dalle altre parti del citoplasma, o dall' ambiente esterno. Modificata la funzione, si modifica pure la struttura di questa parte del citoplasma. Difatti se noi osserviamo la fig. 2 notiamo, che la parte meno fornita di deutoplasma mostra nella sua struttura un aspetto diverso da quella, in cui sono contenuti i granuli di vitello. Ma di più notiamo in questo stadio una disposizione 
del citoplasma evidentemente vantaggiosa per la diffusione delle sostanze liquide, prodotte dalla digestione del dentoplasma; questa disposizione troviamo nelle colonne citoplasmatiche, che circondano le zolle vitelline e si portano con un' apparente struttura fibrillare verso la parte sfornita di deutoplasma. E di.più quest' ultima è disposta prevalentemente verso la parte esterna dell' intestino, vale a dire è più a contatto colle altre parti dell' organismo e colla sua circolazione plasmatica e sanguigna, che ben presto comincia.

Tutte queste disposizioni tendono a favorire il metabolismo citoplasmatico.

Fatti analoghi si hanno nel nucleo.

Anch' esso si dispone in modo da venir più facilmente in contatto con i prodotti della digestione intracellulare, sviluppando la sua superficie in una determinata direzione, in quella cioè secondo eui decorrono - a quanto si giudica dalla struttura - le correnti endoplasmatiche: dall' altra parte esso si avvicina con un estremo verso il limite intestinale più esterno e viene quindi più in rapporto col resto dell organismo.

Ciò avviene dove si ha localizzazione del deutoplasma.

Dove invece esso diminuisce ma non si localizza, tutto il citoplasma deve conservare la sua funzione digerente: e difatti la struttura citoplasmatica, dove il vitello nutritivo è sparso omogeneamente, non appare differenziata in modo apprezzabile, come per esempio nella fig. 3.

Fino a qui noi non abbiamo distinti dei limiti cellulari. Questi compaiono quando il deutoplasma è quasi scomparso del tutto. In questo periodo il metabolismo citoplasmatico non può più avvenire a spese del vitello, perchè questo esiste solo in traccie. La sostanza nutritiva deve quindi esser assorbita allo stato liquido dall' esterno.

Finora dall esterno veniva assorbito solo un corpo gassoso, l'ossigeno, la diffusione quindi si faceva molto facilmente: oppure venivano assorbite sostanze liquide dal solo strato più esterno superficiale del citoplasma. Questo ultimo deve invece ora nella sua totalità assorbire la sostanza liquida dall' esterno. Per rendere più facile l'assorbimento di sostanze difficilmente diffondibili, urge perciò diminuire lo spessore delle pareti che queste devono superare. Si impone perciò un aumento della superficie rispetto alla massa, il frazionamento di questa in tante unità, assorbenti ciascuna da sè, in tante cellule: e nei miei preparati, soltanto adesso, quando solo poche zolle vitelline si scorgono più nell interno del citoplasma, è possibile 
distinguere dei limiti cellulari, e ritenere il citoplasma diviso in tante cellule.

Il citoplasma di queste cellule non ha più nessuna funzione digestiva, esso difatti si vede fornito di una struttura molto diversa, da quando conteneva abbondante il deutoplasma: il suo aspetto è eguale quasi nella sua totalità a quella parte del citoplasma, che prima restò sfornita delle zolle vitelline.

Ma la forma delle cellule è diversa, a seconda della posizione che prima occupava il rispettivo citoplasma ed a seconda delle sue inclusioni.

Dove il deutoplasma era localizzato, come si è detto, e dove il nucleo si era allungato, i limiti cellulari si sono andati formando parallelemente al diametro massimo del nucleo stesso: le ragioni di questo fatto possono stare in fatti di struttura protoplasmatica, o nell'attrazione del nucleo sul protoplasma, o infine nel fatto che cosi il nucleo viene in contatto più diretto coi liquidi nutritivi: forse più d'una di queste ragioni agiscono contemporaneamente. Il nucleo in queste cellule si allunga ancora nella direzione della maggior massa protoplasmatica, creando cosi nuove condizioni al suo assorbimento ed al suo metabolismo.

Nelle cellule che si sono formate intorno ai nuclei, circondati omogeneamente da deutoplasma, e che quindi in tutte le direzioni sono presso a poco egualmente sviluppati, anche il protoplasma ha assunto una forma rotondeggiante, sicchè il nucleo è più uniformemente circondato dal protoplasma. Il piccolo accumulo di citoplasma che nelle cellule glandolari si trova fra nucleo e lume è probabilmente richiesto dalla sua speciale funzione, come cellula di secrezione.

Secondo queste considerazioni adunque è il fatto della modificazione, che avviene nell' assorbimento e nel metabolismo per la scomparsa del dentoplasma, quello che determina il cambiamento di forma, cioè dei rapporti geometrici, degli elementi e la divisione in cellule del citoplasma intestinale. Anche la differenziazione della struttura del citoplasma sarebbe dovuta alla cessazione della sua funzione digerente sul dentoplasma.

Ma un' altro fatto ha in queste ricerche fermata la nostra attenzione, la costanza, cioè, della forma e della grandezza degli elementi, in ciascuno stadio di differenziazione: costanza, che non si mantiene solo nei vari giorni di sviluppo degli embrioni normali, ma si estende anche agli embrioni, che provvenendo solo dalla metà della sostanza embrionale di un novo, dovrebbero tendere ad un risparmio di materiale da costruzione nell' ontogenesi. 
Possiamo mettere questo fatto in accordo colle nostre precedenti considerazioni?

Abbiamo veduto come si debba ritenere, che i rapporti geometrici degli elementi, si vadano modificando per il metabolismo di questi: noi abbiamo veduto l'importanza di questi rapporti e della grandezza per l'assorbimento.

Noi dobbiamo pensare perciò, che qualunque modificazione nella grandezza o nella forma dell elemento debba far sentire la sua influenza modificandone il metabolismo. Se noi pensiamo infatti, che una cellula cresca oltre il volume normale, il ricambio materiale dovrà necessariamente in quella cellula subire, caeteris paribus, un rallentamento, perchè, per la diminuzione di superficie rispetto al volume, l'assorbimento e lo scambio dei materiali del metabolismo sarà reso più difficile; il contrario avverrà se la cellula rimarrà inferiore al volume normale. Difatti noi sappiamo che in genere gli animali - come i mammiferi - che hanno gli elementi cellulari più piccoli, hanno un metabolismo molto più attivo, che non quelli ad elementi più grandi, come gli amfibi. Ma nel caso della cellula che varia dal volume normale, fa d'uopo pensare al fatto, ehe il protoplasma di ogni specie di cellule è adattato ad una determinata qualità e velocità di metabolismo: se questo per il mutato scambio di materiali viene modificato, la cellula dovrà necessariamente andare incontro $o$ all' atrofia o all' esaurimento 0 ad un' altra forma degenerativa, che necessariamente dovrà condurla alla morte. Può però anche avvenire che tale mutato metabolismo porti a qualche modificazione della cellula compattibile colla vita. Questo per il mutamento nella grandezza dell' elemento cellulare o nucleare. Sulle modificazioni della forma, cioè della struttura geometrica, si potranno fare osservazioni analoghe. Difatti in questi casi si viene modificando la posizione delle varie parti della massa dell' elemento, rispetto alla sua superficie; avvicinamento, allontanamento, posizione fra due superficie ecc., ed anche delle varie parti fra loro. Ciò porterà uno squilibrio nel metabolismo delle singole parti e quindi dell' elemento intero, e questo potrà condurre a tutte quelle conseguenze a cui abbiamo accennato ora.

Perciò un cambiamento nella forma o nella grandezza di un elemento, deve necessariamente portare $\%$ la morte o la modificazione dell' elemento stesso, modificazione che potrebbe anche essere incompatibile colla vita dell' organismo intero.

E' per questa ragione, che, quando noi troviamo un elemento 
in un determinato stadio di differenziazione, dovremo altresi vedere, che la sua forma e la sua grandezza sono quelle tipiche, per quella forma di differenziazione istologica.

Prima di raccogliere brevemente, quanto qui è stato discusso, sento il dovere di giustificare, un concetto che per tutte queste pagine si è andato estendendo: il concetto cioè, che l'intestino dei primi stadi di sviluppo, non sia distinto in cellule, ma sia un tutto citoplasmatico indiviso, nel quale si trovano i nuclei; e che, quando il deutoplasma va scomparendo, il citoplasma si divida in tante cellule quanti sono i nuclei, raccogliendosi intorno a questi, come a centro.

Per il fatto che si ammetta una grande massa citoplasmatica unita, con molti nuclei, credo nessuno troverà nulla da eccepire. A parte i protisti polinucleati, noi conosciamo anche negli organismi superiori, degli elementi con un corpo eitoplasmatico unico e molti nuclei, come sono le cellule giganti. D'altra parte è noto, che ci sono condizioni fisiche dell ambiente, che impediscono la segmentazione del citoplasma; cosi Normas ha trovato che la concentrazione dell' acqua di mare impedisce la segmentazione del citoplasma nelle uova di echidni, mentre si segmenta il nucleo: rimesse queste uova nell' aequa di mare normale, il citoplasma si divide in tante cellule, quanti erano i nuclei preformati: avviene appunto quanto avviene, secondo me, negli embrioni di tritone.

Sappiamo anche che l'accumulo di deutoplasma è un ostacolo alla segmentazione del citoplasma, come ci rivela la lenta segmentazione del polo vegetativo, riceo di sostanze vitelline, in confronto del polo animale nelle uova di amfibi.

La segmentazione del citoplasma contenente le zolle di vitello nutritivo deve quindi considerarsi come un lavoro congiunto con grave dispendio di forze. E'naturale quindi che l'organismo risparmi queste forze, quando, non essendo necessario l'assorbimento di sostanze liquide dall' esterno per l'accumulo del vitello, l'intestino non ha bisogno di essere diviso in tante cellule. I nuclei però, che non si trovano in condizioni cosi favorevoli per il loro metabolismo, si devono segmentare. Quando il deutoplasma è consumato, ed il citoplasma ha bisogno di ricorrere per il suo metabolismo, a materiale esistente al difuori dell intestino, diventa necessaria la divisione in cellule. Allora questa divisione si' effettua tanto più facilmente in quanto è scomparsa una delle resistenze più forti a questa segmentazione, l'accumulo di vitello. Il protoplasma si raccoglie intorno ai nuclei, che 
agiscono come centri d'attrazione e che rappresentano, potenzialmente delle cellule anche a citoplasma indiviso.

Dopo questa digressione, raccogliamo, finalmente, quanto crediamo risulti dalle nostre osservazione.

Abbiamo veduto che il eitoplasma rimane integro ed indifferenziato fino a che non ha bisogno di assorbire altro che sostanze gassose dall' ambiente esterno, essendo in esso accumulata la sostanza nutritiva. Il nucleo invece ha bisogno sempre di ricevere dall' ambiente suo la sostanza nutritiva allo stato liquido, per poterla assorbire.

Durante lo sviluppo, mentre si modificano le condizioni del metabolismo del citoplasma, questo si va differenziando: si modifica quindi anche il metabolismo del nucleo, che perciò cambia di forma per raggiungere le condizoni migliori per l'assorbimento.

Quando è quasí scomparsa la sostanza nutritiva che era immagazzinata nel citoplasma e che lo rendeva indipendente quasi dall' ambiente esterno, comincia anche per questo la necessità dell'. assorbimento di sostanze liquide dall' esterno, e quindi si impone l'aumento della superficie di fronte alla massa, la divisione in cellule. Il citoplasma allora si raccoglie intorno al nucleo come a centro e lo circonda, seguendo probabilmente l'attrazione nucleare e disponendosi nel modo più acconcio per il metabolismo della cellula e del nucleo. Questo cambiamento nel ricambio porta per conseguenza una modificazione nella forma del nucleo.

La forma e la grandezza degli elementi determinano la forma e la quantità dell' assorbimento e del metabolismo; una loro modificazione che non sia determinata dalla necessità della differenziazione non può avvenire, per ogni stadio evolutivo, se non portando conseguenze molto gravi, anche per la vitalita dell elemento.

Concludendo potremo raceogliere in alcune brevi proposizioni i punti principali di queste osservazioni, e dire:

1. La divisione in cellule non è necessaria per la vitalità del citoplasma, se non quando essa sia condizione indispensabile per l'assorbimento delle sostanze necessarie al ricambio materiale.

$2^{\circ}$ La modificazione nel metabolismo di un elemento, determinata dalla scomparsa del deutoplasma accumulato nell' uovo, è accompagnata da modificazioni nella forma e nella grandezza dell' elemento stesso e nella struttura istologica di questo.

$3^{\circ}$ La forma e la grandezza degli elementi sono tipici per ogni specie di elementi, nè possono modifiearsi senza grave danno di questi ultimi, modificandosi con essi il metabolismo delle cellule e dei nuclei. 
$4^{\circ}$ Il metabolismo della cellula e del nucleo è legato intimamente alla grandezza ed ai rapporti geometrici, cioè alla forma di questi elementi.

$5^{\circ} \mathrm{La}$ divisione in cellale del citoplasma, la forma e la grandezza degli elementi sono l'espressione - in quanto non lo sieno di una funzione specifica (cellule nervose, fibre muscolari) - delle necessità e delle modalità del metabolismo della sostanza vivente.

Abbiamo in queste pagine seguita la differenziazione degli elementi intestinali, abbiamo visto come la differenziazione istologica sia accompagnata da quella geometrica, cioè della forma e della grandezza, e come tutte due queste differenziazioni sieno dominate dal metabolismo.

Per queste considerazioni e per altre ancora, non crediamo inopportuno, ricercare con una più minuta analisi del fatto dell' ontogenesi, se il metabolismo non abbia per la evoluzione embrionale un significato più ampio e più importante di quello che non gli sia stato fin qui attribuito.

Sarà questo l'argomento della seconda parte di questo studio.

\section{Parte II.}

\section{Sui fattori della differenziazione embrionale.}

I.

\section{Le teorie odierne sulle cause dello sviluppo embrionale.}

Abbiamo veduto nella prima parte di queste ricerche, quale sia l'importanza della grandezza e della forma. della cellula per il metabolismo di questa, e come il metabolismo stesso sia condizione della natura e delle vitalità della cellula. In questa seconda parte vogliamo esaminare, se questo metabolismo abbia un' azione importante nel metamorfismo embrionale. Dovremo perciò ricercare quali sieno i fattori di questo metamorfismo, ed analizzare quindi nei suoi elementi il fatto ontogenetico.

$\mathrm{Su}$ questo argomento i lavori di indole sperimentale e teoretici sono numerosi. Una rivista in italiano dei primi fu già da me fatta, e tralascierò quindi di parlarne, limitandomi qui ad esporre le teorie che sulle cause e le condizioni dello sviluppo embrionale furono fino 
ad oggi emesse. Non sarebbe però possibile esaminare partitamente ciascuna di queste teorie, senza dilungarci troppo dal nostro tema.

Di molte teorie, anche di illustri scienziati, ci sarà forza tacere, perchè ormai già troppe antiche; delle teorie odierne esamineremo solo le più importanti, quelle ehe sono attualmente oggetto della più vivace discussione. Queste sono in principal modo quelle di Hertwig, Roux, Delage, Driesch, Weismann e de Vries: esse sono anche quelle, che rappresentano, in modo più evidente, le tendenze delle varie scuole.

Ma esponiamo partitamente queste varie teorie.

Teoria di Delage. (Teoria delle cause attuali.)

Nell' uovo la distribuzione del citoplasma, che dipende dalle forze molecolari delle varie parti di questo, determina da una parte la posizione del piano di segmentazione, e dall' altra la distribuzione dei materiali alle due cellnle figlie.

Questi sono i due fattori essenziali della differenziazione; dalla posizione dei solehi dipende la differenziazione anatomica, dalla distribuzione del materiale quella istologica. Ma con ciò non si ruol dire, che due cellule non potrebbero dare origine a due tessuti diversi se non avessero ereditato, nascendo, qualche diversità nella loro intima struttura: chè due cellule di identica costituzione possono seguire uno sviluppo diverso a seconda delle condizioni, alle quali sono soggetti.

Nè si deve credere che tutte le sostanze chimiche che servono allo sviluppo dell' embrione si trovino già preformate in esso, come ammette la maggior parte degli autori: molte di queste sostanze possono aver origine per reazioni chimiche nelle cellule, fra i materiali di queste e quelli portati dall' osmosi: queste reazioni non portano solo alla sostituzione della sostanza che si consuma, ma ancora alla formazione di sostanze di altra natura, che saranno diverse nelle diverse cellule (azione morfogena delle ingesta).

Altra azione per la differenziazione dell' embrione è data dalla secrezione interna dei vari tessuti, che stimola o modifica le varie cellule dell' organismo (azione morfogena delle egesta).

Anche le condizioni fisiche dell' embrione hanno un' azione morfogena considerevole, ed una cellula, che per la segmentazione prenda in un organismo un posto determinato, è causa di una quantità indefinita di effetti, che sarebbero stati diversi, se la cellula si fosse situata un poco diversamente. 
Infine, anche le condizioni dell' ambiente esterno esercitano la loro influenza nello sviluppo dell' embrione.

Teoria di de VRIEs.

La teoria'di DE VRIES, che l'autore chiama l'ipotesi della pangenesi intracellulare, non potremmo riferire meglio, che riportando le sue parole stesse:

»Per pangenesi intendo - fatta eccezione dall' ipotesi del trasporto delle gemmule attraverso tutto il corpo - l'idea di DARwin, che ogni carattere trasmissibile per eredità sia legato nella sostanza vivente della cellula ad uno speciale veicolo matẹriale. Questi veicoli chiamo pangeni. Ogni carattere ereditario ha un pangene speciale. In ogni organismo vi sono molti pangeni, e precisamente in tanto maggior numero, quanto più differenziato è l'organismo stesso.

»Per pangenesi intracellulare intendo l'ipotesi che tutto il protoplasma vivente sia costituito da pangeni.

- Nel nucleo sono rappresentati tutti i pangeni di ciascun individuo. Nel resto del protoplasma di ogni cellula si trovano solo quei pangeni, che devono in essa entrare in attività.

»Quest' ipotesi porta alle seguenti conclusioni.

"Ad eccezione di quei pangeni, che sono attivi già nel nucleo, p. e. quelli che dominano la segmentazione nncleare, tutti gli altri devono uscire dal nucleo per diventare attivi. Ma la maggior parte di questi rimane nel nucleo, dove si divide in parte per la divisione nucleare, in parte per l'emigrazione nel protoplasma. I pangeni, trasportati dalle correnti plasmatiche, giungono negli organi protoplasmatici, dove si uniscono con i pangeni già esistenti, si moltiplicano ed entrano in attività. Tutto il protoplasma consta dunque di pangeni usciti a varie riprese dal nucleo, e dei loro derirati: non esiste un' altra sostanza vivente fondamentale. «

\section{Teoria di Driesch.}

Secondo Hans Driescri lo sviluppo ontogenetico si deve considerare nel modo seguente.

Nell' novo fecondato sono contenute poche specificità protoplasmatiche (anisotropia del plasma), mentre al contrario, il nucleo si deve considerare come indifferente (isotropia del nucleo).

Nella segmentazione dell' uovo le diversità plasmatiche sarebbero divise nelle varie cellule, mentre i nuclei di queste rimarrebbero totipotenti (capaci di dare origine a qualsiasi parte dell' organismo). 
Le cause dei processi morfogeni, possono essere divise in tre gruppi di fenomeni elementari:

$1^{0}$ Funzioni elementari delle cellule o processi morfogeni elementari.

$2{ }^{\circ}$ Fattori che agiscono liberando forze di tensione raccolte nell'organismo (Auslösungsfaktoren), agendo cioè come cause occasionali. Questi fattori sarebbero localizzati e determinerebbero così la sede del loro effetto.

$3^{\circ}$ Il fatto del ritmo evolutivo, il succedersi, cioè, ordinato e costante dei singoli processi ontogenetici.

Le funzioni elementari della cellula sarebbero secondo RAUber (e Driesch si associa alla classificazione di questo autore), l'aumento numerico, l'acerescimento, la migrazione e la differenziazione delle cellule.

Specialmente l'accrescimento per assorbimento d'acqua e per assimilazione, la migrazione e la secrezione cellulare hanno una grande importanza morfogena.

Esistono poi i fenomeni morfogeni propri al corpo vivente, quale corpo fisico e sistema meceanico: fra questi sono da notare: la correlazione di massa (Rovx), la capillarità, e l'osmosi.

I processi elementari sono da ricondurre a processi sia fisici, sia chimici elementari, o almeno sono da considerare come introdotti da tali processi. Il risultato dell' ontogenesi avviene per fasi chimiche. Questi processi chimici sono da considerare come fattori liberatori di forze di tensione.

Ogni processo morfogeno elementare è liberato da uno stimolo che agisce sul nucleo; il protoplasma è l'intermediario chimicamente specifico fra la causa "liberatrice" ed il nucleo, ed è da ritenere quale zona di percezione dello stimolo, mentre il nucleo ne sarebbe la zona d'azione. Quando il nucleo entra in azione, lo stimolo non determina una scissione di materiale specifico per il processo elementare che si sta svolgendo, ma al contrario il nucleo conserva la sua totalità. Questa apparente contraddizione si elimina, ammettendo che i materiali determinanti ciascun processo elementare non provvengano dal nucleo, ma si originino nel protoplasma sotto la direzione del nucleo, direzione che è da ritenere come un' azione fermentativa. Il nucleo è, secondo Driesch, un miscuglio di sostanze simili a fermenti, di cui ciascuno rappresenta una specie di processo elementare dell ontogenesi in azione. Per un processo di liberazione di energia latente viene posto in azione un determinato fermento, che può 
essere un fermento specifico, perchè il corpo plasmatico, che funge come zona di percezione, possiede un chimismo specifico.

Quando viene posto in azione un materiale nucleare, il corpo cellulare, che prima influiva su di esso, viene modificato sotto la sua direzione, e cosi vien posto il fondamento per un nuovo processo elementare, che è alla sua volta percettore di nuovi stimoli per l'avvenire.

Cosi la causa liberatrice si manifesterebbe in ogni processo morfogeno dapprima sul nucleo, lo modificherebbe; questa modificazione porterebbe, dopo la segmentazione, una modificazione del chimismo delle cellule figlie.

La struttura specifica di tutti i nuclei di un' organo uniforme, in via di sviluppo, è nguale fino a che l'organo sia compinto ed entri in azione un nuovo stimolo. I nuclei di ciascuna formazione sono eguali, finchè è eguale il carattere istochimico delle rispettive cellule.

Quanto agli stimoli, che agiscono in ciascuna modificazione ontogenetica, questi sarebbero soltanto spinte, momenti occasionali che producono effetti sproporzionati, ineguali, agli stimoli stessi, per qualità e quantità, essendo latenti nel corpo stesso sia la qualità dell' effetto, sia l'energia necessaria a produrlo.

Gli stimoli poi sono dati da induzioni di massa, che dipendono dalla presenza di materiali diversi nel germe; da induzioni chimiche, dovute all azione di sostanze chimiche, proprie di un organo, su altri organi, azione che si esercita forse sotto la forma di fermento. Fra queste induzioni chimiche sono da annoverarsi anche le induzioni di direzione, cioè la chemiotassi. Altri stimoli sono dati dalle induzioni dell' ambiente esterno e dagli adattamenti funzionali, messi dapprima in luce da Roux, che ne determinò la causa nella lotta delle parti nell' organismo.

Quanto al ritmo dell' ontogenesi, è da notare, che i processi morfogenetici danno prodotti che hanno una durata limitata. Questi processi possono essere primari (formazione dell' entoderma e dell' ectoderma nei vertebrati) o secondari (come p. e. lo sviluppo del tubo midollare) o terziari, quartari ecc., (sviluppo delle cinque vescicole cerebrali, della vescicola oftalmica ecc.) ed è necessario che sia chiuso un processo precedente perchè ne possa sorgere uno seguente. Ultimari sono infine quei processi che sono caratterizzati dal non dar luogo a nessun altro processo morfogenetico. A ciascuno di questi processi è propria una speciale struttura istochimica. 
Questa è in breve riassunto la teoria di DrIEsch per quanto riguarda la parte biologica: non possiamo in questi cenni seguire l'antore nella parte puramente filosofica del suo lavoro, e nelle sue digressioni teleologiche, perchè usciremmo dal compito nostro. Ci limiteremo quindi alla disamina critica di questa prima parte della teoria analitica di DrIesch.

\section{Teoria di Hertwig.}

Secondo questo antore lo sviluppo ontogenetico non si può ritenere dipendente, causato e regolato dalla sostanza embrionale (idioplasma); ma bisogna considerare le cause che lo producono, come poste al di fuori di questa sostanza, per quanto vengano prodotte, per opera del processo evolutivo in regolare successione. Queste cause risiederebbero, in primo luogo nei rapporti reciproci in cui pervengono fra di loro le cellule di un organismo, mentre aumentano di numero in seguito alla segmentazione: in secondo luogo poi nell' azione dell' ambiente esterno.

Questi due gruppi di cause; interne ed esterne, non si possono però assolutamente separare le une dalle altre, perchè le cause esterne, che influiscono su di un periodo evolutivo, passano a far parte, in un periodo successivo, delle cause interne.

Potremo perciò dire che la ineguale differenziazione delle cellule, è la reazione della sostanza organica a stimoli ineguali.

Una delle fonti principali per lo sviluppo di differenziazioni è il segmentarsi delle cellule. Per il fatto che la sostanza nucleare assimila mano a mano, per una serie di complicati processi chimici, il materiale accumulato nell' uovo e l'ossigeno dell' atmosfera, si hanno delle differenziazioni sempre maggiori. Perchè l'accrescimento della sostanza nucleare produce una divisione della stessa in $2,4,8$, 16 porzioni ecc., le quali separandosi e distribuendosi in varie parti dell' uovo, vanno a costituire nuovi centri individuali di energia, intorno ai quali la sostanza dell' uovo si aggruppa e si trasforma in cellule.

Ogni accrescimento poi è congiunto colla tendenza ad un aumento della superficie, per rendere possibili i processi di assorbimento, sicchè avviene che le cellnle si dispongano in fili, membrane ecc. Su questo fatto si fonda la diversità della organizzazione delle piante e degli animali: essa si spiega per l'antagonismo che esiste nel loro modo di assimilazione. Nelle piante lo sviluppo di superficie è verso l'esterno perchè da questo assorbono il materiale nutritivo, negli animali al contrario verso l'interno. 
L'aumento numerico delle cellule porta delle differenziazioni, anche per il fatto, che ciascuna di esse, pur rimanendo eguale alla cellula-uovo non rappresenta più il stutto*, ma solo una parte del \tutto\%. "Essa dunque non è più indipendente, ma è una parte subordinata di una unità più elevata, e, come tale, viene influenzata nelle proprie funzioni dall' organismo intero e dalle altre cellule: e ciò tanto più, quanto più aumenta il numero delle cellule, fino a tanto che l'autonomia della cellula, come organismo elementare, è talmente ridotta, che essa appare solo come una parte che funziona in dipendenza del tutto.

Negata cosi l'individualità della cellula si deve ammettere che la direzione del suo sviluppo ulteriore non dipenda da cause ad essa intrinseche, ma dai rapporti suoi coll' organismo intero e colle sue parti, e da quelli con l'ambiente esterno: rapporti che naturalmente cambiano, a seconda del posto occupato dalla cellula nella compagine dell' organismo; la differenziazione diversa delle cellule è perciò una funzione della loro sede.

Per azioni esterne diverse, da uno stesso substrato embrionale si possono sviluppare prodotti molto vari, come viene dimostrato dai fatti di dimorfismo e polimorfismo, dovuti a diversa nutrizione della larva.

Ma quale è la parte, che al processo evolutivo prende l'idioplasma stesso?

E qui da notare, che- la cellula reagisce in un modo specifico agli stimoli che la colpiscono; e agli stessi stimoli i vari organismi possono reagire in modo diverso: diversità che non può dipendere che dalla differente natura dei vari organismi, cioè dalla loro struttura micellare.

Si deve ammettere perciò, che lo sviluppo ontogenetico prenda le mosse, da una sostanza embrionale già altamente e specificamente organizzata: ma a questa sostanza non si possono attribuire che quelle proprietà, che sono inerenti al carattere della cellula, e non già proprietà, che possono essere prodotte per l'unione di molte cellule, sotto l'azione di condizioni esterne.

Ma la differenziazione polare bilateralmente simmetrica dell' novo determina la direzione dei solchi di segmentazione, la natura e la grandezza delle cellule, e la sede dei futuri processi evolutivi. Perciò l'uovo è da considerare come una forma a cui l'embrione, che se ne sviluppa, si deve fino a un certo segno adattare per molti riguardi. E nulla dice che le cellule di ogni distretto, che si può discernere nell' uovo rappresenti la sostanza già differenziata dei 
vari organi; al contrario si deve sostenere, che solo all' ulteriore progresso dello sviluppo sia riservato di stabilire quello, che da ogni cellula debba derivare.

La teoria di Hertwig sarebbe perciò, secondo l'antore, preformista, in quanto ammette, come punto di partenza dell' evoluzione embrionale, un idioplasma specifico; epigenista, in quanto considera, quali fattori delle varie differenziazioni cellulari - in cui si compendia lo sviluppo ontogenetico - agenti estranei alla sostanza embrionale stessa, quali sarebbero: l'accrescimento della sostanza nucleare, con susseguente anmento del numero dei nuclei e delle cellule: la correlazione fra $i$ vari elementi cellulari e fra $i$ vari organi: infine le azioni dell' ambiente esterno, sotto forma di nutrizione, temperatura ece.

Quanto ai problemi speciali dello sviluppo embrionale, la teoria lascia il campo aperto a ricerche e studi per ogni caso speciale.

$\grave{\mathrm{E}}$ ancora da ricordare che HERTwIG in altro luogo, mentre sostiene, che nella segmentazione delle cellule la sostanza ereditaria viene divisa ugualmente nelle due cellule figlie, aggiunge di accordo con NaEgelr, che ogni cellula dell' organismo è capace per il suo idioplasma di diventare germe di un nuovo individuo: se questo poi si avvera o no dipende dal plasma nutritivo (di NaEgeli). Osserva ancora l'autore che tutti i nuclei di tutti i tessuti (fatte poche eccezioni) dello stesso organismo, per struttura e per grandezza sono presso a poco eguali: affermazione alla quale contraddicono recenti studi molto numerosi.

\section{Teoria di Roux 1 ).}

Numerose sono le esperienze di questo autore, e le interpretazioni, che di ciaseuna di esse egli ha dato, si collegano in modo da darci un edifizio teoretico, che qua cerchiamo di riassumere.

Nell' uovo fecondato dobbiamo tener conto di due fattori, l'idioplassone contenuto nel nucleo, e le sostanze vitelline. Il primo è la sostanza ereditaria, la sostanza che imprime la sua fisonomia alla

1) La teoria di Roux, o per meglio dire le varie teorie, che questo autore ha esposte, si trovano sparse in numerosi lavori: riesce perciò difficilissimo, raceoglierle in poche pagine, come un thtto organico. In questa esposizione abbiamo tenuto conto, specialmente di tutte le rettifiche, che Roux ha fatto seguire alle critiche mosse alle sue teorie. Speriamo perciò di aver evitata ogni inesattezza, e se cio non ci fosse riescito, ne chiediamo venia all' autore. 
cellula vivente. In esso sono contenuti tutti i caratteri dell' individuo, che se ne svilupperà. Nella segmentazione l'idioplassone si divide in modo ineguale nei nuclei figli: in uno di questi si trasporta il materiale ereditario di un certo numero di caratteri, nell' altro, quello di altri caratteri: la segmentazione del nucleo è qualitativamente ineguale (erbungleiche Theilung). Una certa parte però dell' idioplassone, si trasmette immutata ad ambedue le cellule figlie, senza però, che in queste entri in attività (idioplassone accessorio, Reserveidioplasson), il che avviene solo in determinate circostanze. Questa segmentazione ineguale avviene già nella prima divisione cellulare, che separa, nella rana, la metà destra dalla metà sinistra del futuro embrione, corrispondendo il primo solco di segmentazione al piano mediano.

I quattro primi blastomeri nelle uova di rana si sviluppano ciascuno per proprio conto (autodifferenziazione) e sono indipendenti l'uno dall' altro, per quanto riguarda i loro discendenti: lo sviluppo dell' uovo di rana si deve quindi considerare, come un lavoro di mosaico, costituito per lo meno di quattro pezzi, che si sviluppano, ciascuno indipendentemente dall' altro (teoria del mosaico). Le cellule che derivano da uno di questi blastomeri, probabilmente sono coordinate tra loro nello sviluppo, potendo ciascuna cellula dare l'impulso a processi evolutivi in un' altra (Aulösungsfaktoren).

Questo avviene nello sviluppo tipico o normale dell' organismo. Esiste però uno sviluppo atipico, nella rigenerazione e nella postgenerazione dell' autore.

Quando l'equilibrio delle sostanze vitelline venga turbato, queste agiscono, mercè il loro disorientamento, sul nucleo, mettendo in attività l'idioplassone di riserva, che in ogni nucleo si trova, agendo come stimolo che trasforma una forza di tensione in forza viva (Auslösung). Questo idioplassone di riserva dà poi l'indirizzo allo sviluppo ed alla natura della cellula. Le sostanze vitelline perciò, darebbero l'impulso allo sviluppo cellulare, ma questo sarebbe regolato dal nucleo.

Questa è nei suoi tratti principali la teoria di Roux, ehe non possiamo qua esaminare nei particolari.

\section{Teoria di Weismann.}

La teoria esposta da questo geniale zoologo non è soltanto una teoria sulla differenziazione embrionale: essa e piuttosto una teoria dell' ereditarieta. Come tale essa tratta e dei processi per i quali 
vengono accumulate nelle cellule sessuali le qualità paterne e materne che si trasmettono ai figli, e del modo in cui da queste cellule sessuali, che si sono fuse per l'atto della fecondazione, si sviluppi un' organismo complicato, che riproduce i caratteri propri dei genitori. Una parte della teoria Weismanniana si compendia nella »continuità del plasma germinativo « e nella immortalità di questo: noi non possiamo però fermarci su questo interessante tema, perchè esso sconfina dai limiti di queste ricerche, e passiamo perciò a dire brevemente di quella parte dell' ipotesi di Wersmans, che riguarda i processi dell' evoluzione ontogenetica.

Weismann distingue nell' organismo pluricellulare due specie di sostanza vivente, il ssomatoplasma , che costituisce la parte vitale delle varie cellule del corpo, ed il »germiplasma« o plasma germinativo, che è raccolto nel nucleo delle cellule sessuali, e serve alla trasmissione dei caratteri ereditari. Continuamente nell' evoluzione ontogenetica si sviluppa dal plasma germinativo il plasma somatico, per un processo molto complicato di segmentazione assimetrica, la quale forma il caposaldo di tutta la teoria. Per segmentazione assimetrica - come io mi permetto di tradurre l'espressione di serlungleiche Theilung* - intende WeISManN il fatto da lui supposto, che in tutte le segmentazioni cellulari (come ammetteva dapprima), $o$ in quelle segmentazioni cellulari, che preludiano ad una formazione di nuovi organi o a differenziazioni di cellule (come ebbe a dire più recentemente), il plasma germinativo si distribuisce in modo diverso, sia per quantità che per qualità, nelle due cellule figlie. Perchè, secondo questo autore, il plasma germinativo avrebbe una struttura straordinariamente complicata. Esso si comporebbe di un grande numero di elementi chiamati ide, che si uniscono a gruppi detti idanti: questi idanti poi a volte sarebbero visibili nei cosidetti cromosomi. Le ide alla lor volta sarebbero costituite da elementi di ordine inferiore, i determinanti, ehe sarebbero composti di un grande numero di biofori o portatori della vita i quali rappresenterebbero le vere unità biologiche come le »unità fisiologiche * di Spencer. Per comprendere il significato biologico di tutte queste unità di vario grado, immaginate dallo zoologo di Freiburg, dobbiamo ricordare che egli ammise, che nell' novo fecondato si trovino già preformate tutte le qualità, le diversità, i caratteri che sono propri all' organismo, che da questo uovo si svilupperà. Ora tutti questi caratteri si trovano raccolti nelle varie ide. Avremo perciò un ida per ciascun gruppo di cellule, tessuto organo o altro. Il carattere 
di ogni cellula è preformato nel determinante, e quando questo entra in azione si scinde nei suoi biofori che, venendo sparsi nella cellula relativa, ne determinano la natura. Nelle segmentazioni dell'novo e dell' embrione e più precisamente in' quelle segmentazioni che introducono una differenziazione cellulare, le singole ide vengono distribuite diversamente nelle varie cellule: cosi nelle segmentazioni che precedono la formazione della gastrula, le ide degli organi ectodermali verranno trasmesse alle cellule, che danno origine all ectoderma, le ide invece p. e., del fegato alle cellule entodermali. Cosi a poco a poco le singole ide si isolano ed $i$ loro determinanti si distribuiscono nelle varie cellule, di cui determinano la natura per mezzo dei biofori. Cosi il plasma germinativo si trasforma in plasma somatico. Questo non è più capace di riprodurre l'organismo da eui provviene. Non peró tutto il plasma germinativo subisce questa trasformazione: una parte ne rimane inalterata e va a costituire le cellule sessuali del nuovo organismo. Di più è da notare che anche le altre cellule, che l'autore dice cellule somatiche, per distinguerle da quelle sessuali, ricevono oltre alle ide ed ai determinanti propri e che ne determinano la natura, anche ide e determinanti che normalmente non si sviluppano, restando allo stato latente, e costituiscono l'idioplasma accessorio: questo solo in determinate circostanze può rivelare la propria presenza, imprimendo i suoi propri caratteri alle cellule in cui alberga.

Da questo breve riassunto che delle teorie sulle cause dello sviluppo embrionale, siamo andati facendo, si è veduto, che a due concetti opposti sono informate le varie teorie. In alcune di esse si ammette, che nell' uovo sieno già preformate tutte le sostanze e tutti i caratteri, propri a ciascuna specie ed a ciascun individuo: l'uovo sarebbe una miniatura dell' organismo già sviluppato, mentre quest' ultimo sarebbe la proiezione della struttura del primo. Questa teoria della preformazione, che $i$ tedeschi con parola impropria chiamano dell' evoluzione, si ritrova negli scritti di Roux, Weismann, e DE VRIEs ed inoltre in quelli di Nussbaum, Wagner ed altri. Le altre teorie, quelle di Hertwig, Delage e Driesch, sono epigeniste, secondo esse, cioè, nell' uovo fecondato, non ci sono che pochi caratteri e poche sostanze in confronto di quelli dell' embrione che se ne svilupperà. Da questa struttura relativamente semplice si vanno man mano, nel corso dell' ontogenesi, sviluppando e diffe- 
renziando le particolarità del nuovo organismo, per una serie di processi, determinati da cause inerenti in parte all uovo stesso, in parte a fattori esterni. Epigenisti sono ancora oltre agli autori citati, specialmente Herbst, HAaCke, Wilson ed altri.

Quale di queste due tendenze, di questi due concetti, considereremo come più giustificato? E quale delle teorie proposte si accorda più con le idee di fisiologia generale oggi dominanti, e corrisponde più ad una retta interpretazione dei fatti osservati?

Per rispondere a questi quesiti, ci fa d'uopo esaminare brevemente nei suoi elementi il processo ontogenetico, per potere valutare alla stregua dei fatti queste si diverse teorie.

II.

\section{Critica delle teorie.}

Il principio fondamentale sul quale si basano le teorie preformiste di Roux e Weismann, è costituito dall' ipotesi che nella segmentazione delle cellule embrionali la sostanza ereditaria, l'idioplasma, venga trasmesso in modo ineguale alle cellnle figlie. Questa ipotesi ha avuto origine dalle esperienze di Roux del 1888. Egli, pungendo con un ago caldo nno dei due primi blastomeri di un uovo di rana, otteneva, dal blastomero rimasto intatto, un mezzo embrione. $\mathrm{Ne}$ trassero gli autori la conclusione, che da un blastomero solo poteva prender origine un mezzo embrione soltanto, perchè ogni blastomero conteneva solo la metà del materiale ereditario di tutto l'uovo, e precisamente il materiale della metà destra o di quella sinistra del corpo. Ma ben presto altri ricercatori poterono constatare nelle uova di echinodermi, meduse, ascidie, ctenofori, amphioxus, teleostei ed infine anche degli amfibi stessi, che da una metà dell'uovo, quando questa sia isolata, si sviluppa non mezzo embrione, ma un embrione intero e normale in tutto, tranne che nella grandezza.

Ma v'ha di più. Driesch ha dimostrato nelle uova di echidni, e Hertwig in quelle di amfibi, che si ottiene sempre un embrione in complesso normale, anche se, mediante una moderata compressione, >si gettano sossopra i blastomeri, come un nucchio di palle «, per modo che $i$ blastomeri vengono a dar origine ad organi diversi, da quelli che ne nascerebbero nello sviluppo normale.

Perciò non si può ammettere, che nei vari blastomeri sia contenuta sostanza ereditaria diversa, perchè in tal caso non potrebbero dar origine che ad un determinato organo o tessuto. 
Per non dover abhandonare l'idea della segmentazione a trasmissione ereditaria ineguale, si ricorse allora all' altra ipotesi dell'idioplasma accessorio. Si ammise cioè che ad ogni nucleo venga trasmessa una parte sola della sostanza ereditaria in forma attiva, ma, contemporaneamente, anche una parte dell' idioplasma completo, che però non funziona ma rimane allo stato latente.

Quando perỏ qualche turbamento avviene nello sviluppo normale (disorientamento delle sostanze vitelline, secondo Roux), questo agisce come stimolo sull' idioplasma accessorio, ed una o l'altra parte di esso entra in attività, dando origine ad uno sviluppo, che Roux chiama atipico.

Tralasciando di parlare dell' opportunità di introdurre questo nuovo concetto nell' ontogenesi, diremo solo, come sembri poco probabile, che un disorientamento nella sostanza vitellina, comunque esso avvenga, metta sempre in attività quella tal parte di idioplasma accessorio, che proprio necessita per ottenere uno sviluppo normale; al contrario sarebbe molto più facile, che lo sviluppo di questo idioplasma accessorio, per stimoli tanto diversi, prendesse le vie più diverse, e portasse ad organismi nel più vario modo mostruosi.

Per ovviare a questa difficoltà, Weismann ha negli ultimi tempi ammesso, che la segmentazione a trasmissione ereditaria ineguale non avrenga in tutte le segmentazioni, ma solo in quelle che preludiano alla formazione di nuove differenziazioni.

A parte la circostanza che nessun fatto esiste, che provi questa asserzione, esistono dei fatti che la negano assolutamente: cosi prineipalmente il fenomeno dell' eteromorfosi. Di più noi crediamo, che un' attenta ricerea della differenziazione cellulare di alcuni organi, debba portare luce non indifferente su questo argomento.

Noi dobbiamo quindi assolutamente rigettare ogni teoria, che si fondi sulla divisione ineguale dei materiali ereditari nella segmentazione nucleare; dobbiamo quindi respingere ogni teoria preformista: contro la quale però parlano ancora altri fatti, così il dimorfismo di alcuni insetti, cosi tutta la teratologia.

Noi dovremo pereiò ricercare in una teoria prevalentemente epigenista l'interpretazione del fatto dell' ontogenesi, della differenziazione o del metamorfismo degli elementi.

Ma quale delle teorie fino ad oggi proposte soddisfa di più alla nostra ragione e si accorda meglio coi fatti conosciuti, e con le idee generali di fisiologia che oggi abbiamo?

Esaminiamo partitamente le tre teorie epigeniste, più su esposte, 
Ricerche sulla differenziazione cellulare nello sviluppo embrionale.

quella di Driesch, quella di Hertwig e quella di Delage. E cominciamo da quella di Driesch.

Quali sono i punti principali di questa teoria?

Da poche diversità del plasma dell' uovo si va sviluppando un numero sempre maggiore di differenziazioni. Queste avvengono per processi di liberazione di energia latente, provocati da stimoli, che, agendo sul nucleo, lo modificano non stabilmente: il nucleo agisce allora quale fermento sul corpo plasmatico e lo modifica stabilmente. Avviene perciò che alla fine dell' ontogenesi il corpo cellulare ha una struttura specifica, mentre il nucleo conserva la sua totipotenza. Il nucleo poi non avrebbe una determinata struttura fisica, ma sarebbe un miscuglio di molti fermenti. I processi morfogenetici avvengono per processi cellulari elementari, quali l'accrescimento, la differenziazione ecc.

Campeggia in questo quadro, che il Drirsch ci fa delle canse dello sviluppo embrionale, l'ipotesi, che ogni fatto ontogenetico avvenga per la liberazione di forze latenti. Questa asserzione, per quanto non appoggiata da fatti, potrebbe avere un valore teoretico, qualora potesse da sè rischiarare qualche punto oscuro dello sviluppo embrionale, e se non avesse bisogno, per essere sostenuta, dell' appoggio di altre numerose ipotesi, di cui alcune in parte non bene accordabili coi fatti conosciuti.

Queste ipotesi sono: la mancanza di struttura fisica nel nucleo e l'uguaglianza e la totipotenza di tutti i nuclei. Basta ricordare $i$ numerosi lavori di citologia, che hanno messo in rilievo la struttura dei nuclei delle diverse cellule, per dichiarare inacettabile quest' ultima asserzione di Driescr. La struttura istologica dei nuclei delle cellule nervose, specialmente studiate in un recentissimo lavoro, mostrano tante e tali diversità dai nuclei di altri tessuti, da rendere impossibile ogni errore in proposito. E DrIESCH stesso ammette, che la diversità della struttura istologica dipenda da una diversità nella struttura chimica.

Altra ipotesi che da nessun fatto viene appoggiata è quella, che il nucleo sia un miscuglio di vari fermenti. Nessuna osservazione fino ad ora autorizza una simile deduzione.

Infine Driesch ei parla di processi cellulari elementari, per cui avvengono i fatti organogenetici. Questi processi, come abbiamo veduto, sono l'aumento numerico delle cellule, l'accrescimento, la migrazione e la differenziazione. Che questi processi non sieno per nulla elementari è facile dimostrare; essi non sono che la risultante 
di altri processi più semplici, che posssono essere elementari o anche non esserlo. Cosi l'aumento numerico delle cellule è la conseguenza del processo di segmentazione, quando questo aumento numerico non sia accompagnato dall' accrescimento degli elementi stessi; chè se questo ha luogo l'aumento numerico è la risultante di due processi più semplici, l'accrescimento prima, la segmentazione dopo. Ma che nè l'accrescimento, nè la segmentazione sieno processi elementari è cosa troppo ovvia: la segmentazione è preparata da cambiamenti strutturali così complicati che ciascuno di questi costituisce da sè stesso un processo più semplice e per nulla elementare: cosi i mutamenti della parte cromatica, cosi la formazione del fuso, la segmentazione dei centrosomi ecc. L'accrescimento poi alla sua volta è conseguenza di fatti vari e di processi più semplici come l'assorbimento, l'assimilazione, la respirazione. Nè altro vale della migrazione attiva delle cellule.

Veniamo infine alla differenziazione. Anche questa non può essere chiamata un processo elementare: al contrario esso è uno dei processi più complessi, determinato da cause ancora ignote. Ed è appunto queste cause che andiamo ricercando nello studio dei fattori dello sviluppo ontogenetico: infatti il processo dell' embriogenesi riposa in gran parte sulla differenziazione delle cellule. Ma se le cause della differenziazione sono ancora da ricercare - non persuadendoci quelle proposte da Driesch - pure abbiamo dati sufficienti, per le cognizioni di fisiologia generale e per alcuni studi di citologia, per asserire che la differenziazione cellulare è la risultante di altri processi più semplici come l'assimilazione, l'assorbimento, l'accrescimento ecc.

Della teoria di Driescr possiamo perciò accettare soltanto la parte generale, che considera l'ontogenesi, come lo sviluppo di molte diversità da una materiale dapprima poco differenziato: e ad accettare questa proposizione siamo indotti dai noti fatti di embriologia sperimentale, specie quelli riguardanti lo sviluppo di blastomeri isolati, dai fatti dell' eteromorfosi, da alcuni fatti patologici (cisti dermoidi) dai fatti del dimorfismo ecc. Ma per quanto riguarda la spiegazione di questo fatto, per quanto concerne l'interpretazione dell'epigenesi, la mancanza di prove di fatto, che dimostrino l'attendibilità dell' ipotesi di Driesch, il grande numero di ipotesi accessorie (che in parte mancano pure di una base di osservazioni, in parte sono contrarie ai fatti conosciuti), ci obbligano a ricercare in altri fattori la causa della differenziazione embrionale.

Passiamo alla teoria di Her'rwig. 
Il concetto che in questa predomina è, che l'uovo non può contenere preformati in sè, che quei caratteri, i quali sono propri alla natura di cellula, e non altri. I caratteri propri ad un organismo complesso, devono formarsi man mano nello sviluppo ontogenetico: e ciỏ succede per vari fattori, non intrinseci alla struttura dell' idioplasma, ai quali questo reagisce in una determinata maniera. Fra questi fattori troviamo, l'assimilazione, la segmentazione, lo spostamento delle cellule. Date però condizioni esterne diverse, si possono sviluppare prodotti molto diversi da uno stesso substrato.

Queste conclusioni di HerTwig sono quelle che più di tatte le altre teorie tengono conto dei fatti generali della fisiologia: mentre gli altri autori non considerano l'embrione che si evolve, soggetto alle stesse leggi fisiologiche degli altri organismi viventi, ed immaginano per esso strutture e processi che non hanno base nei fatti accertati, nè riscontro negli altri fenomeni biologici, HerTwig non invoca altre leggi che quelle comuni a tutti i viventi, ed i fatti meccanici più manifesti, che si succedono nell' embrione. Cosi tra i primi l'assimilazione, la respirazione, tra i secondi gli spostamenti di cellule. Ad ogni modo, per lui le cause determinanti lo sviluppo embrionale, non sono intrinseche all' idioplasma, ma agiscono su questo, per modo che lo svilnppo ontogenetico è da ritenersi, come la reazione della sostanza vivente a stimoli esterni; ed è appunto come reazione del plasma organizzato all' ambiente in cui si trova, che noi consideriamo ogni fenomeno vitale.

Per queste considerazioni crediamo che si debba accettare la concezione sull' ontogenesi, da HERTwiG formulata. Essa però riguarda solo il principio generale, che in questo processo domina, nè cerca di specificare quali sieno in particolare le singole cause dello sviluppo degli organismi. A far ciò tende più la teoria di Delage, che del resto non si scosta nei suoi principi, da quella dell' anatomico berlinese.

Difatti ambedue ammettono una determinata struttura dell' novo come fondamento e principio dell' ontogenesi, ambedue ritengono, che il numero delle differenziazioni vada aumentando durante le sviluppo embrionale. Delage poi crede, che sieno specialmente la direzione dei solehi di segmentazioni e la distribuzione delle sostanze dell' uovo quelle, che determinano la differenziazione; poi, da queste stesse cause occasionate, la formazione di nuove sostanze chimiche, l'azione morfogenetica della secrezione interna, la struttura fisica dell'embrione ecc. Ora noi dobbiamo riconoscere che tutti questi fattori, 
che hanno un' azione non sconoscinta nei processi vitali degli organismi adulti, debbano logicamente sussistere ed avere una determinata influenza anche nel processo evolutivo.

Noi pertanto, di tutte le teorie proposte per risolvere l'intricato problema delle ragioni dello sviluppo embrionale, non possiamo ritenere consone a guanto ei è noto dalla fisiologia generale degli esseri viventi, che la teoria di HerTwIG per il principio evolutivo generale e quella di Delage per quanto cerca dì applicare più da vicino questo principio stesso.

Ma tutte due queste teorie mentre stabiliscono un principio irrefutabile, che ci deve guidare nella ricerca del scome e del "perchè dell' evoluzione embrionale, ed accennano ai fattori possibili di questa, non determinano invece i fattori reali di tale processo.

Crediamo che sulla scorta di questi principi, ei sia dato di accostarci un po' di più, ad una fruttuosa ricerca di tali fattori, tenendo conto dei risultati della prima parte di questo studio, e facendo tesoro di quanto la fisiologia e la biologia generale hanno ormai acquisito, quale patrimonio sicuro, alla nostra conoscenza dei processi vitali.

\section{III.}

\section{Sull' importanza del metabolismo nella differenziazione embrionale.}

Che cosa s'intende, o per meglio dire, in che consiste la differenziazione embrionale?

Delage la distingue in differenziazione anatomica e differenziazione istologica, intendendo per la prima la disposizione degli elementi nell' embrione, per la seconda la struttura degli elementi. Questa nomenclatura non ci pare molto esatta; meglio sarebbe chiamare la seconda differenziazione citologica. Ma anche in questo modo il processo metamorfico non è bene analizzato nei suoi elementi.

DrIEsch, abbiamo veduto, scinde questo processo in 4 processi elementari, che, come si è detto, non si possono considerare come tali.

Noi possiamo ritenere esser cinque i fatti che compendiano lo sviluppo, o la differenziazione che dir si voglia, dell' organismo dall'uovo; ma questi fatti che sono simili ai "processi elementari" di DrIesch, son per noi processi biologiei già molto complicati.

Essi sono:

$1^{0}$ Modificazione del numero degli elementi.

$2^{\circ}$ Modificazione della grandezza degli elementi. 
$3^{\circ}$ Modificazione della forma degli elementi.

$4^{\circ}$ Modificazione della posizione degli elementi.

$5^{\circ}$ Modificazione della struttura degli elementi.

Esaminiamo ora quali sono realmente i processi elementari che servono di base a queste modificazioni.

$1^{\circ}$ II numero degli elementi cellulare può aumentare o diminuire. Il caso più frequente nello sviluppo embrionale è naturalmente il primo, ma non è escluso che anche il secondo possa avverarsi (sviluppo regressivo degli organi rudimentali: timo nel primo tempo della vita estranterina; coda nei girini di rana). L'aumento può essere unito ad una modificazione della grandezza degli elementi 0 no. Si può quindi arere:

a) aumento del numero degli elementi, con aumento della loro grandezza,

b) aumento del numero degli elementi, con diminuzione della grandezza,

c) aumento del numero degli elementi, rimanendo invariata la loro grandezza.

Nel primo caso, nella segmentazione di una cellula, le cellule figlie riacquistano il volume della cellula madre e lo oltrepassano di una piccola quantità. Nel secondo caso le cellule si segmentano senza che le cellule figlie raggiungano la grandezza della cellula madre: in alcuni casi esse rimangono eguali alle metà di quest' ultima (formazione delle cellule di segmentazione). Nel terzo caso, in fine, le cellule figlie, dopo (o durante) una segmentazione, riacquistano la grandezza precisa della cellula madre (riproduzione comune delle cellule).

Lasciando per ora da parte la modificazione della grandezza, occupiamoci dell' aumento numerico delle cellule. Questo viene iniziato da un complicato processo nell interno del nucleo, processo che porta ad una grande modificazione sebbene passeggera nella struttura di questo elemento. Questo processo cariocinetico, in cui tatti ammettono un grande dispendio di energia, deve esser legato a processi chimici di varia natura, specialmente esotermici. Cosi si sa che nella germinazione delle piante, in cui attivissima è la segmentazione, si ha uno sviluppo di calore apprezzabilissimo.

La segmentazione è quindi da considerarsi come la manifestazione istologica di processi chimici, che avrengono precipuamente nel nucleo, come conseguenza, cioè, del suo metabolismo.

Cosi pure la diminuzione del numero delle cellule, che può 
esser la conseguenza della loro morte e del loro riassorbimento, dipende evidentemente da modificazione della struttura chimica di queste cellule.

Scindere il processo del metabolismo in processi più elementari, non è possibile, senza uscire dal campo della biologia ed invadere quello della chimica e della fisica.

Perciò diremo: il processo elementare, che determina la modificazione del numero delle cellule e dei nuclei in un organismo, che si sta sviluppando, è il metabolismo di questi elementi istologici.

$2^{\circ}$ La grandezza di un elemento può diminuire o aumentare: la diminuzione può dipendere:

a) dalla segmentazione cellulare non seguíta da una sufficiente ricostruzione del materiale;

b) dal consumo non riparato, del materiale cellulare durante la funzione di questo.

L'aumento invece, da un esagerata produzione,

a) del materiale proprio della cellula,

b) di materiale diverso da quest' ultimo.

Di questo ultimo modo di produzione ci occuperemo al $5^{\circ}$ punto.

La diminuzione della grandezza dell' elemento istologico, se è legata unicamente alla segmentazione della cellula, dipende, come si è dimostrato al punto $1^{\circ}$, dal metabolismo della cellula. Se essa poi dipende da consumo del materiale cellulare non riparato convenientemente, dovremo spiegare questa diminuzione con dei fatti distruttivi della materia vivente, con processi chimici di ossidazione, con processi catabolici.

Il contrario vale per l'aumento di volume, dipendente da formazione di nuovo materiale cellulare. Il processo elementare è qui un processo chimico di sintesi, un fatto anabolico.

In ambedue $\mathrm{i}$ casi perció, nella diminuzione e nello aumento della grandezza cellulare, sono i fatti chimici distruttivi e reintegrativi, che insieme formano il metabolismo della cellula, quelli che costituiscono il processo biologico elementare.

$3^{\circ}$ La forma di una cellula è regolata dalla pressione che domina nel suo interno e da quella che subisce dalle altre cellule. Due sono i gruppi di fattori che concorrono a stabilire tale pressione:

a) l'accrescimento e la tensione della cellula stessa,

b) la grandezza, la quantità e la disposizione delle cellule vicine.

Quanto all' accrescimento della cellula stessa rimandiamo a quanto s'è detto al punto precedente: ma anche la tensione interna 
di una cellula (che nelle cellule animali difficilmente dipende da sola imbibizione) è doruta alla formazione di nuovi liquidi nell' interno della cellula per opera del plasma già esistente; è dovuta quindi a processi chimici, sia che si immaginino sotto la forma di assimilazione, sia sotto la forma di secrezione.

L'altro fattore della forma delle cellule è la vicinanza delle altre cellule: la grandezza ed il numero di queste abbiamo veduto esser l'espressione di processi metabolici della sostanza vivente. Ciò, come vedremo al punto seguente, vale anche per la disposizione delle cellule.

Anche la forma delle cellule dipende quindi da processi elementari, che si estrinsecano come fatti progressivi o regressivi del metabolismo cellnlare.

$4^{\circ}$ La posizione di nna cellnla, può variare per attività di questa o per azioni esterne alla cellula.

Lo spostamento attivo si può avere

a) perchè la cellala cresce in una determinata direzione,

b) perchè la cellula si segmenta e le cellule figlie si spostano in una certa direzione,

c) per la migrazione della cellula.

Che i due primi modi di spostamento attivo sieno l'espressione del ricambio materiale, non fa mestieri dimostrare, dopo quanto si è detto più indietro.

Ma si può dire lo stesso anche della migrazione delle cellule: essa consiste in fenomeni di movimento, di attività funzionale della cellula, che non si possono concepire se non ammettendo, che la cellula utilizzi delle energie di tensione, che si trovano nella sua trama istochimica, e le trasformi in forza viva, sotto l'aspetto di movimento: il che vuol dire che la cellula per muoversi scinde in composti più semplici, più ossidati, le sostanze di cui essa è formata. Anche la migrazione perciò è da considerarsi come l'espressione del metabolismo, e precisamente del eatabolismo della cellula.

Sarebbe inutile insistere sul fatto, che alla stessa stregua si debbano giudicare anche gli spostamenti passivi di una cellula, dovati all accrescimento del numero e del volume, della forma e della posizione delle cellule vicine.

$5^{\circ}$ Abbiamo reduto che i processi, per i quali si compie l'ontogenesi, e che fino ad ora abbiamo analizzato, sono l'espressione di un solo processo veramente elementare, il metabolismo, sia progressivo, che regressivo.

Potremo applicare questo principio, anche al fatto più impor- 
tante dell' ontogenesi, alla differenziazione istologica dei tessuti e delle cellule?

Che le diversità istologiche tra due cellule, riposino prevalentemente su diversità chimiche, nessuno vorrà negare, per quanto anche altri fattori come la struttura micellare ecc. possono avere una certa influenza. Si deve però anche ritenere che esistano diversità chimiche fra cellule di eguale struttura istologica, diversità che si riflettono nell' attivita vitale della cellula, senza che sieno apprezzabili direttamente con i nostri mezzi di indagine.

Ma queste diversità si devono ammettere, che esistano già nell'uovo fecondato: ed allora il metabolismo non avrà nessuna azione sul metamorfismo della sostanza vivente: esso non farà che riprodurre ed aumentare le diversità chimiche già esistenti senza crearne di nuove. Oppure si deve ammettere che queste diversità si formino durante l'ontogenesi. In tal caso il metabolismo non dà origine solo a materiale uguale a quello esistente, ma ne crea continuamente di differente.

Quale di queste due possibilità possiamo noi accettare?

Noi abbiamo veduto nella prima parte di queste ricerche, come, mentre si trasforma il metabolismo di una cellula, si inizi in essa una modificazione nella forma già solo per obbedire alle esigenzè dell' assorbimento. Noi sappiamo dalla fisico-chimica, come le condizioni fisiche, la forma dell' ambiente in cui un reazione chimica si compie, bastino a modificare questa reazione, ed a permetterne $o$ no lo svolgimento. Perciò anche solo per il fatto che il metabolismo determina la forma della cellula, esso può portare un cambiamento nella struttura istologica della medesima. Ma di più abbiamo visto, che il cambiamento nel metabolismo porta delle modificazioni dirette nella struttura di una cellula, e che da una stessa sostanza vivente, a seconda del vario metabolismo, si possono ottenere cellule di diversa struttura e di diversa funzione.

Ma una prova diretta che nell' ontogenesi si formino delle sostanze di composizione chimica diversa dalla sostanza già vivente, è stata fornita fin dal 1888 da Luciani e Piutri. Questi autori hanno dimostrato, che nelle uova del bombice del moro in via di sviluppo, il quoziente respiratorio non è rappresentato da una costante, ma da un valore che cresce progressivamente fino a superare l'unità.

Ciò dimostra all' evidenza, che in queste uova, che si stanno sviluppando, c'è la formazione di molecole chimiche diverse per struttura da quelle già esistenti, molecole chimiche meno ossigenate, 
fornite cioè, di una somma di energia potenziale sempre più considerevole.

Così è chiaramente dimostrato, che non tatte le sostanze chimiche si trovano già preformate nell' uovo, ma che esse si vanno formando durante l'ontogenesi.

Il metamorfismo, abbiamo detto, dipende specialmente da formazione di sostanze chimiche nuove, e dalle condizioni fisiche della cellula: forma, grandezza ecc., condizioni tutte, che abbiamo veduto esser l'espressione di un solo processo elementare, del metabolismo.

Sicchè alla fine di questa disamina dei fattori, in cui si scompone il fatto dell' ontogenesi, arriviamo alla conclusione, che essi non sono per nulla processi biologici elementari, ma che, come ogni fatto vitale, sono esponenti di un solo processo elementare, dello scambio chimico delle molecole viventi, o, come diciamo più brevemente, del metabolismo.

E percio se vogliamo comprendere il processo dell' ontogenesi, è nostro compito studiare come questo metabolismo si comporti e come si comporti rispetto ad esso la materia vivente.

Già nella prima parte di queste ricerche, abbiamo veduto, che la grandezza e la forma della cellula modificano le condizioni del metabolismo, e che appunto perciò esse sono costanti per ciascuna specie di cellule. Ora poi, abbiamo veduto che forma e grandezza sono dipendenti alla lor volta dal metabolismo.

L'ontogenesi ci appare perciò come una serie di processi metabolici, e gli effetti di un processo metabolico sono la causa di un processo metabolico seguente. Si ha perciò una catena di fatti intimamente legati fra loro.

Cerchiamo di seguire queste successioni.

Nel metabolismo di ogni cellula noi dobbiamo distinguere da una parte la struttura chimica ed istologica della cellula stessa, dall'altra i materiali, che entrano a prender parte al processo metabolico. E' chiaro perciò che quest' ultimo è l'espressione del modo in cui una data sostanza vivente reagisce a stimoli chimici che la colpiscono.

Perciò bisogna ritenere, che ciascuna sostanza vivente deve avere un metabolismo proprio, e diverso da quello delle altre sostanze, per ciascuno stimolo. D'altra parte è pure evidente che a stimoli chimici diversi una stessa sostanza reagirà con metabolismo differente.

Da ciò risulta, che da ciascun uovo, in cui esiste un determinato idioplasma, dovrà derivare un organismo diverso, avendo ciascuno 
uno speciale metabolismo. E d'altra parte non occorre ritenere ciascun idioplasma, specifico per un dato individuo, perchè lẹ diversità che sempre esistono fra gli stimoli che colpiscono due vova anche identiche, bastano per spiegare le differenze individuali minime, che si riscontrano in due organismi.

Questo per la parte che nel metabolismo prende la sostanza vivente. Quanto all' azione che in esso hanno le sostanze non organizzate, questo puó dipendere da parecchi fattori: dalla quantità di questi materiali, dalla qualità loro, dalla rapidità e dalla direzione in cui si succedono nella cellula, dalla loro pressione osmotica ed altri molti. Questi materiali si trovano al principio dell' ontogenesi, in generale, immagazzinati già nell' novo in una determinata quantità e disposizione, e sono sempre gli stessi per ciascuna specie. Di più in ciascuna specie di animali le uova hanno la stessa grandezza e la stessa forma. Data quindi questa identicità nelle condizioni, in cui entra nel metabolismo la sostanza non organizzata, e data l'eguaglianza dell' idioplasma, è chiaro perchè sia sempre eguale lo sviluppo delle nova di ciasenna specie di animali, dovendo in essi esser sempre eguale il metabolismo, per ciascun grado di sviluppo.

Al principio dell' evoluzione embrionale, il metabolismo è tale, da portare solo al processo di segmentazione, senza che si abbia un accrescimento successivo delle cellule figlie. Questo continua per un certo tempo, ma presto si mutano le condizioni del metabolismo: per il continuo aumento numerico degli elementi, non accompagnato da un accrescimento di volume, sono mutati molto i rapporti di grandezza delle cellule e dei nuclei e sono perciò modificate le condizioni di assorbimento e di diffusione dei materiali nutritivi; di più le cellule per il reciproco contatto vanno mano mano cambiando di forma, ed i nuclei, per le segmentazioni, si spostano dalla loro sede primitiva, e vengono a trovarsi nelle più varie sezioni dell' uovo. Tutto ciò deve modificare potentemente il metabolismo dell' idioplasma, sicchè questo incomincia anche a dar vita a nuova sostanza vivente, simile o no a quella già esistente; le cellule, cioè, riacquistano dopo la segmentazione il loro volume primitivo, ed in alcuni casi incominciano anche a differenziarsi chimicamente o istologicamente.

Queste nuove modificazioni nella natura della sostanza vivente, devono far si, che essa debba reagire nel suo metabolismo in modo diverso che per l'innanzi, anche se gli stimoli rimangono eguali: tanto più poi, se questi mutano.

Per tutto ciò il metamorfismo deve acquistare dei gradi sempre 
più elevati, e trovandosi le varie cellule in condizioni diverse, si avrà oltre al metamorfismo generale, una differenziazione diversa nelle diverse cellule.

Quando poi cessano di esistere nell interno dell' uovo i materiali nutritivi, questo fatto segna un tale mutamento nel metabolismo, che noi abbiamo potuto seguire al microscopio il metamorfismo che ne segue.

Dunque riassumendo, per noi il fatto ontogenetico è l'espressione del metabolismo di un dato idioplasma: questo metabolismo porta man mano ad un certo numero di differenziazioni, le quali modificano alla lor volta questo metabolismo ed ogni mutamento di quest' ultimo implica, come sua conseguenza, nn nuovo metamorfismo. Questa azione reciproca di metabolismo e metamorfismo continua cosi, fino alla completa differenziazione dell' organismo. Sono perciò precipui fattori della differenziazione embrionale, tutte quelle cause che agiscono in modo da portare qualche cambiamento nel metabolismo cellulare. Questi fattori possono essere estranei all' uovo, o possono a questo essere intrinseci e dipendere dal fatto ontogenetico stesso: e mentre sono consegtuenza di un processo di sviluppo embrionale, sono la causa del processo seguente. Tali sono la forma, la grandezza, la posizione delle cellule embrionali, ed altri, che qua non vogliamo più da vicino esaminare.

Questo mgdo di concepire l'embriogenesi, ci pare pienamente giustificato, perchè tenendo conto dei fatti speciali, che in essa si avverano, non dimentica che essa è pure un fenomeno biologico, e perciò soggetto alle stesse leggi di tutti gli altri processi biologici. Noi abbiamo ricordato, che oggi un processo vitale non si può concepire, che come ricambio della molecola proteica e perciò abbiamo considerato come tale anche l'ontogenesi. Ma inoltre questa ipotesi ha in suo favore, all' infuori dei fatti ricordati nelle pagine precedenti, anche i fatti del dimorfismo delle api, delle formiche e delle termiti, che, come ognuno sa, sono dovuti alla diversa nutrizione delle uova di questi insetti. Ed in appoggio ancora parla tutto quello, ehe noi sappiamo sul ricambio materiale dell' embrione. Ma altri fatti si spiegano con quest' ipotesi nel modo più semplice.

In primo luogo notiamo il ritmo nei processi ontogenetici. Questa regolare successione degli stadi ontogenetici, che nessuna teoria preformista riesce a spiegare in modo positivo, appare una necessaria conseguenza delle nostre premesse. Perchè avvenga un dato processo morfogenetico, è necessario che il plasma organizzato reagisca con un 
dato metabolismo agli stimoli, è necessario quindi, che esso abbia una determinata struttura e che gli stimoli sieno di una data natura; ed ambedue questi fattori dipondono dal metabolismo, che le generazioni precedenti di cellule hanno subito. E' chiaro perciò che ciascun processo può solo evolversi, quando si sieno già svolti tutti quei processi, che hanno dovuto preparargli le condizioni necessarie per essere; vale a dire, che ciascun processo debba presentarsi in un determinato momento' ed in una determinata sede.

Un altro problema, che affatica da qualche anno i biologi, è l'interpretazione dello: sviluppo dei blastomeri isolati. Il fatto, che da questi si ha un embrione intero, mentre quando essi rimangono aderenti all' altro blastomero, anche morto, danno origine solo a mezzo embrione, si può spiegare ammettendo, che i processi metabolici, non portino ad un metamorfismo molto importante durante la formazione dei primi solehi di segmentazione. La differenziazione avviene più tardi, e sarà diversa se il blastomero è isolato o no, perchè nel primo caso esso rappresenta un uovo intero per $i$ suoi rapporti geometrici, ed il suo metabolismo decorre come in un uovo intero: nel secondo caso invece i suoi rapporti di forma e posizione mutano molto, ed esso non può dare che un mezzo embrione.

Un' obbiezione si potrebbe sollevare.

Come mai se i processi ontogenetici dipendono dal metabolismo, e questo alla sua volta è influenzato da tanti fattori esterni, il prodotto dell' ontogenesi è quasi costantemente lo stesso?

Risponderemo, che in primo luogo, i prodotti dell ontogenesi non sono eguali mai perfettamente, anche quando si tratti di due discendenti della medesima coppia; in secondo luogo, che nel caso, in cui il metabolismo decorra per una causa qualsiasi in modo molto atipico, si hanno $o$ i prodotti teratologici, o addirittura l'arresto di sviluppo. Nel caso poi, in cui questa anomalia fosse ancora maggiore, si ha un arresto di sviluppo cosi precoce, che noi non veniamo neppure a conoscenza del processo ontogenetico iniziato.

Ciò però non può avverarsi con molta frequenza, perché esistono alcune disposizioni, che regolano questo metabolismo. Nei primi momenti dell' ontogenesi, infatti, come abbiamo notato più su, per l'identicità dell' idioplasma, delle sostanze nutritive, della grandezza e della forma dell' novo, il metabolismo è determinato nel modo più stretto. Più tardi la sostanza vivente è già più differenziata, e sempre più perciò si limita il modo in cui può reagire ad un certo stimolo. Difatti una cellula embrionale ha la massima libertà nel modo di 
Ricerche sulla differenziazione cellulare nello sviluppo embrionale. 101

reagire ai vari stimoli: cosi come hanno dimostrato HerTwig e Driesch, dipende dalla posizione della cellula la sua destinazione futura. Una cellula differenziata invece non può reagire che in un dato modo: cosi una cellula ossea reagirà a qualsiasi stimolo, meccanico, chimico o funzionale, solo colla formazione di nuovo tessuto osseo.

Sicchè, quando le condizioni esterne del metabolismo si cominciano a fare più varie, ed a perdere di determinatezza, è la maggior differenziazione della cellula, quella che impedisce che il processo evolutivo possa seguire una via troppo anormale.

Cosi siamo giunti alla fine di queste considerazioni che s'accostano molto da vicino alle teorie di Hertwig e di Delage, e nelle quali abbiamo tentato di accordare i concetti sull' ontogenesi con quelli generalmente ammessi per la fisiologia generale. Possiamo ora raccogliere queste nostre vedute in una frase, dicendo, che la differenziazione embrionale di un organismo è funzione del suo metabolismo.

All' illustre Professore Luciani, che, accordandomi generosa e cortese ospitalità nel suo laboratorio, mi diede opportunità a compiere queste ricerche, esprimo qua la mia sincera gratitudine.

Roma, Laglio 1897.

\section{Zusammenfassung,}

I.

1. So lange der Nahrungsdotter in der Darmwandung des Tritonenembryos in großer Menge vorhanden ist, findet keinerlei Differenzirung der betreffenden Elemente statt, und man kann keine Grenze zwischon den Zellen nachweisen. Das Cytoplasma scheint noch nicht in Zellen gesondert za sein.

2. Mit dem Beginne des Schwindens des Nahrungsdotters fängt die Differenzirung an und wenn sich noch nur sehr wenig Deutoplasma vorfindet, sind die Zellen von einander gesondert.

3. Während der Differenzirung gestalten sich die Zellen und Kerne so, dass der Stoffwechsel am loichtesten vor sich gehen kann.

4. In jeder Form oder Stufe der histologischen Differenzirung sind die Größe der Elemente und die Verhältnisse zwischen den Durchmessern derselben, also ihre geometrische Form, unveränderlich, welches anch das Alter des Embryo sei. - Umgekehrt ändert sich bei jeder histologischen Differenzirung auch die Größe und die Form der Elemente. 
5. Auch in Embryonen, die sich aus einer isolirten ersten Blastomere entwickelt haben, sind die Größe und die geometrische Form der Elemente in jedem Differenzirungszustand ganz die gleichen wie bei den normalen Embryonen in den entsprechenden Differenzirungsstadien.

II.

Die Ontogenese kann als in fünf Processe gesondert angesehen werden:

Veränderung der Zahl der anatomischen Elemente,

Veränderung der Größe derselben,

Veränderung der Form derselben,

Veränderung der Lage and

Veränderung des histologischen Baues (Differenzirang) derselben.

Diese Processe sind aber keineswegs elementare Processe, sondern sie sind alle besondere Erscheinungen eines einzigen elementaren Processes: des Stoffwechsels (Metabolismus), sei dieser syntetisch (Anabolismus) oder analytisch (Katabolismus). Die Differenzirung eines werdenden Organismus, d. h. seine Ontogenese, muss desshalb als die Funktion seines Stoffwechsels betrachtet werden.

\section{Bibliografia.}

Delage, Y., La structur du Protoplasma et les théories sar l'hérédité et les grands problèmes de la biologie générale. 1895. Paris.

de Vries, H., Intracellulare Pangenesis. 1889. Jena.

Driesch, H., Analytische Theorie der organischen Entwickelung. 1894. Leipzig. Galeo'Tti, G., Über die Granulationen der Zellen. Mit 2 Tafeln. 1895. Internationale Monatsschrift f. Anatomie u. Physiologie. Bd. XII.

Herlitzka, A., Sullo sviluppo di embrioni completi da blastomeri isolati di nova di tritone (Bibliografia dei lavori sperimentali fino al novembre 96). Con una tavola. 1897. Archiv f. Entwickelungsmechanik. Bd. IV. Heft 4.

Hertwig, 0., Die Zelle und die Gewebe. 1892. Jena.

Lo stesso, Zeit- und Streitfragen der Biologie. I. Präformation oder Epigenese? 1894. II. Mechanik und Biologie. 1897. Jena.

Lo stesso, Beiträge znr experimentellen Morphologie and Fntwickelnngsgeschichte. I. Die Entwickelung des Froscheies unter dem Einfluss stärkerer und sehwächerer Kochsalzlösnngen. Mit 3 Tafeln. 1895. Archiv f. mikr. Anatomie. XLIV.

LEVI, G., Ricerche citologiche comparate sulla cellula nervosa dei vertebrati. Con due tavole. 1897. Rivista di patologia nervosa e mentale. Vol. II. Fase. V.

Luclani e Piutti, Sui fenomeni respiratori delle nova del bombice del moro. 1888. Atti della R. Accademia dei Georgofili. Firenze. Vol. XI.

Lugaro, E., Modificazione delle cellule nervose nei diversi stati funzionali. 1895. Lo Sperimentale.

Norman, W., Segmentation of the Nuclens without segmentation of the Protoplasma. With 2 plates. 1896. Archiv f. Entwickelungsmechanik. Bd. III. Heft 1. 
Ricerche sulla differenziazione cellulare nello sviluppo embrionale. 103

Nussbaum, F., Einige Bemerknngen im Betreff der Entwickelnngatheorie von Oscar Hertwig. 1895. Biologisches Centralblatt. Bd. XV.

Roux, W., Gesammelte Abhandlungen über Entwickelungsmechanik der Organismen. Bd. II. 1896. Leipzig.

Lo stesso, Über die verschiedene Entwickelung isolirter erster Blastomeren. 1894. Archiv f. Entwickelnngsmechanik. Bd. I. Heft 4.

Lo stesso, $\mathrm{Za} \mathrm{H.} \mathrm{Driesch's} \mathrm{>} \mathrm{Analytische} \mathrm{Theorier.} \mathrm{1896.} \mathrm{Archiv} \mathrm{f.} \mathrm{Entwicke-}$ lungsmechanik. Bd. IV. Heft 3.

Lo stesso, Für unser Programm und seine Verwirklichung. Archiv f. Entwickelungsmechanik. Bd. V. Heft 1 a. 2.

Lo stesso,. Über den Antheil von »Auslösungen* an der individuellen Entwickelung. 1896. Archiv f. Entwickelungsmechanik. Bd. IV. Heft 2.

WAGNER, F. von, Einige Bemerkungen zu 0. HerTwIG's Entwickelungstheorie. 1895. Biol. Centralblatt. Bd. XV.

Weismann, A., Das Keimplasma. Eine Theorie der Vererbung. 1892. Jena.

Lo stesso, Änßere Einflüsse als Entwickelungsreize. 1894. Jena.

Lo stesso, Germinal Selection. 1896. Jena.

\section{Spiegazione della tavola IV.}

Fig. 1. Sezione di intestino indifferenziato.

Fig. 2. Sezione di intestino nel primo stadio di differenzazione (I ${ }^{a}$ forma di differenzazione).

Fig. 3. Sezione di intestino nel $I^{o}$ stadio di differenzazione ( $I^{a}$ forma).

Fig. 4. Cellale intestinali di rivestimento.

Fig. 5. Lo stesso.

Fig. 6. Cellule glandolari dell' intestino. 